\title{
25 Research Soure

\section{Channel structure selection in a competitive supply chain under consideration of marketing effort strategy}

\section{Mingxia Li ( $\nabla$ nicemingxia@sina.cn )}

Nanjing University of Aeronautics and Astronautics https://orcid.org/0000-0001-7329-321X

Kebing Chen

Nanjing University of Aeronautics and Astronautics

\section{Research Article}

Keywords: Supply chain management, channel structure selection, marketing effort, game theory, strategic effect

Posted Date: February 28th, 2022

DOI: https://doi.org/10.21203/rs.3.rs-1147409/v1

License: (c) (1) This work is licensed under a Creative Commons Attribution 4.0 International License.

Read Full License 


\title{
Channel structure selection in a competitive supply chain under consideration of marketing effort strategy
}

\author{
Mingxia $\operatorname{Li}^{1 *}$ and Kebing $\mathrm{Chen}^{2 \dagger}$ \\ ${ }^{1 *}$ Department of Mathematics, Nanjing University of Aeronautics and Astronautics, \\ Nanjing, 211100, Jiangsu, China. \\ ${ }^{2}$ College of Economics and Management, Nanjing University of Aeronautics and \\ Astronautics, Nanjing, 211100, Jiangsu, China. \\ *Corresponding author(s). E-mail(s): nicemingxia@sina.cn; \\ Contributing authors: kbchen@nuaa.edu.cn; \\ †The author contributed equally to this work.
}

\begin{abstract}
We study the optimal decisions of a supplier in terms of channel structure selection and marketing effort strategy when facing a competitor offering substitutable products. By employing game theoretic models for different retail competition scenarios, we show that equilibrium channel structures are primarily determined by the intensity of retail competition along with the marketing effort level. Channel structure selection and marketing effort strategy are interdependent and interactive. Our results show that the supplier has an incentive to make marketing effort and sell products directly to consumers in most cases. When the retail competition becomes fiercer, the supplier can benefit from selecting a distribution strategy based on customers' sensitivity to the supplier's marketing effort. In contrast, the supplier selling products through the downstream retailer will not make marketing effort under certain market conditions (e.g., low competition and high customer sensitivity to the supplier's effort performance), while the competitor adopts the direct-sales strategy. Generally, the supplier will prefer making marketing effort relative to undertaking product distribution. Finally, we also examine the strategic effects of channel structure selection and marketing effort decisions on the competitor's profit in the retail competition market.
\end{abstract}

Keywords: Supply chain management, channel structure selection, marketing effort, game theory, strategic effect

\section{Introduction}

Internet-based commerce has created large marketing opportunities for suppliers to sell to customers directly. Some brands that were previously only available in supermarkets can be purchased online. For example, Coca-Cola and Pepsi both sell their products directly to consumers on JD.com (Wei et al. 2020). In order to further improve competitive advantages, suppliers are motivated to make more marketing efforts. The supplier can increase the sales of products by boosting brand reputations and engaging in promotion and advertising campaigns. These activities signify the supplier's marketing effort (Ke and Jiang 2021, Ma et al. 2017) and can be seen everywhere, whether online or in department. Inman, a 
women's apparel brand, for example actively and constantly attracts consumers through some new media approaches, such as public account tweets, Taobao live and microblog marketing. The sales of all Inman's physical stores reached RMB 20 million in May 2016 (CKGSB 2017). Some milk suppliers (Erie and Mengniu) offer their products to some large retailers (Wal-Mart and Vanguard) in China and they furnish marketing effort (manpower and material resources) to maximize their own profits (Liu et al. 2020). These efforts that occur at the sales stage eventually become the cost, which is generally borne by the supplier. Hence, the supplier should make a careful trade-off before determining the marketing effort level.

Analyzing this trade-off to address whether and when the supplier has a motivation to adopt a direct-sales or distribution channel structure is a main objective of our paper. In a competitive business environment, suppliers have several operational choices to gain advantages, including supply chain integration and product distribution channels. These business options are widely used and provide new opportunities for industries. In addition, suppliers have been very successful at capturing the benefits of distribution strategies by increasing their flexibility, agility and responsive speed. One successful example is Dell, which has always been known for its consistent direct-sales mode. Although this mode allows for a competitive advantage in retail pricing, it weakens communication between suppliers and consumers. In contrast, through a distribution strategy by which Dell sells her products to the downstream reseller Wal-Mart, Dell not only improves the quality of service to customers in the sales process, but also reduces the risks of interferences by nonmarket factors (Morris et al. 2005). Nonetheless, the distribution channel strategy still has some downsides. For example, it can lead to high inventory or high financial risk level (Chen et al. 2020). At the same time, distribution amplifies the negative effect of double marginalization on channel performance. Therefore, suppliers should trade off between benefits and disadvantages before they choose a channel structure.

Our study is motivated by practical considerations for suppliers who have opportunity to employ marketing effort and channel structure strategies in view of the competition in a supply chain. From a sales channel perspective, if a supplier sells her products through a downstream retailer (i.e., the distribution strategy), they form a two-stage decentralized supply chain. In this case, the supplier serves as the leader of the Stackelberg game while the retailer serves as the follower. In addition, a decentralized channel structure could make supply chain decisions more complex, especially in a competitive supply chain environment. In this research, we will identify the conditions a supplier should adopt a distribution strategy in a competitive market and study how a firm's distribution strategies affect its rival's decisions and profits. In addition, if a supplier uses a direct-sales strategy, the supply chain structure is centralized. We investigate retail competition between two partially substitutable products and the demand for each product depends on whether the marketing effort is adopted. We will explore how the supplier decides on channel structure and marketing effort and how this affects profits. To be specific, our work attempts to answer the following questions:

(i) What is the optimal channel structure for a supplier in terms of marketing effort level, competition intensity and profitability? And is there an equilibrium channel structure?

(ii) How does marketing effort level affect a supplier's channel structure strategies and profits? Whether and when does the supplier have an incentive to make marketing effort?

(iii) Considering retail competition, what are the strategic impacts of a supplier's motivations to make marketing effort and select a channel structure on a competitor's decisions and profits?

The main contribution of our paper lies in the extension of the existing studies by exploring a supplier's channel structure selection and marketing effort strategy in the context of price competition. In order to study the impact of marketing effort on the supplier's and her competitor's profits, we design two kinds of demand functions: one with marketing effort provided by the supplier and the other without marketing effort. Subsequently, we consider different channel structures for each demand context: (1) both the supplier and competitor adopt the distribution mode; (2) neither the supplier nor her competitor adopts the distribution mode; and (3) one of the two parties does not adopt the distribution mode while the other does. We derive the equilibrium solutions for all combinations of the different demand 
functions and channel structures and explore the relationships between channel structure and marketing effort strategies. Specifically, if the supplier chooses to make marketing effort for selling the product, the resulting larger demand will lead to an increase in profitability, yet the supplier's effort performance will damage the competitor's profitability under certain market conditions. In addition, a supplier who sells products directly to consumers is likely to benefit more from her marketing effort than a supplier who sells through a downstream retailer. Finally, we investigate how the supplier's channel structure and marketing effort strategies affect its profits and the competitor's decisions.

In the following, we review the literature to identify the research gap and to position our research.

\subsection{Supply Chain Competition}

Many researchers have explored retail competition between two supply chains. An earlier study on competing chains can be found in the work of McGuire and Staelin (1983), which concludes that if two supply chains compete fiercely, operating in a decentralized pattern is the dominant strategy. In recent years, the topic of supply chain competition remains hot. For example, Adida and Demiguel (2011) show the competition of multiple suppliers and retailers in a supply chain and give the unique equilibrium solution. ? consider a two-echelon supply chain with horizontal competition between retailers and vertical competition between a supplier and two retailers. The contracts of wholesale price and revenue-sharing in a decentralized supply chain are also examined to make competitive suppliers reach optimal profit (Ai et al. 2012). Our paper considers two competitive supply chains and each chain is consisting of only one supplier or one supplier and one retailer depending on supplier's channel structure selection. Besides, Fang and Shou (2015) explore the impact of supply uncertainty and competition intensity on equilibrium decisions of order quantity, contract offering, and centralization choice in a chain consisting of a retailer and a supplier. Our research focuses on the distribution channel structure strategy to mitigate retail competitiveness when considering customer's marketing effort sensitivity level. Other various issues on in supply chain competition can be found in research on topics such as corporate social responsibility (Lee et al. 2018), contract choice game (Li et al. 2013), reverse channel choice (Wu and Zhou 2017), factor-market rivalry (Ellram et al. 2013), and strategic sourcing (Chen and Guo 2014).

In most operations management literature on supply chain competition, retail competition has been widely used in the market demand model (Feng and Lu 2013, Hosseinimotlagh et al. 2019, Zhang et al. 2021). Different from their models, our demand function can be influenced by the marketing effort level. The similar assumption can be found in Ma et al. (2017), Zhu and He (2016) and Jamali and Rasti-Barzoki (2018). We study the supply chain competition issue by considering the supplier's motivation for adopting the marketing effort and explore how the channel structure strategy of the supplier affects the rival's decisions, profit and channel structure strategy. In addition, we provide the analytical results on how to choose the strategies of channel structure and marketing effort, whereas most of the existing literature uses a numerical simulation approach (Ghosh and Shah 2014).

\subsection{Channel Structure Selection and Sale Efforts}

Our research is closely related to the supplier's channel structure selection. Due to fierce market competition, the distribution channel strategy design has been studied extensively. Cachon and Harker (2002) consider a distribution strategy where the supplier is playing as a follower while the downstream retailer playing as a leader, and find that the brutal price competition can be reduced effectively in economies of scale. Furthermore, Atkins and Liang (2010) compare both distribution mechanisms to obtain a more general explanation for why decentralization can be preferred for competitive supply chain. Zhao and Shi (2011) study the channel strategy of competitive supply chains with multiple suppliers and a single retailer. Their results show that the decentralization structure is better when competition is fierce, while the centralization structure is better when there are multiple vendors. Our paper also considers the supplier's marketing effort/service strategies when she is faced with a competitor 
offering partially substitutable products. According to the research by Yang et al. (2019), a monopolistic seller's optimal pricing decisions can be affected by selling effort and the direct impact of selling effort is to induce more customers to purchase in the first period. But they do not take into account the competitor's selling effort strategies. Xia et al. (2019) examine service level and distribution channel decisions with competing supply chains and they focus on how service competition affects the channel structure. Actually, marketing effort strategy has been widely applied in dualchannel supply chain. For example, Dan et al. (2014) compare and analyze the optimal service levels in a single-channel supply chain and a dualchannel supply chain, respectively. And $\mathrm{Li}$ and $\mathrm{Li}$ (2016) analyze the pricing policies of two players in a dual-channel supply chain, considering the value-added services to the product and fairness concerns of the retailer. The firm's optimal innovation effort (Li et al. 2020) and wholesale pricing strategies are investigated along with both innovation and advertising contribute to the product demand under different game structures (Song et al. 2017). However, the existing literature does not consider whether the supplier should provide the market effort. In contrast, we discuss these questions, including, when does the supplier furnish the marketing effort in the competitive market and which sales channel does she provide the marketing effort to. From the perspective of the profit, we investigate the impact of channel structure decision and marketing effort level on the competitor and explore the existence of channel structure equilibrium.

To summarize, our paper contributes to the existing literature as follows. First, we extend the research of competitive supply chain issues to the discussion about motivations of the supplier's marketing effort and product distribution. Second, we focus on the effects of the supplier's motivation of effort performance and strategy of channel structure on the competitor's decisions and profit. Third, our paper contributes to the current supply chain management literature by investigating the effects of different channel structure selection of two suppliers on the retail prices and the marketing effort levels. We offer some new insight to the related literature (Jamali and Rasti-Barzoki 2018) as follows: (i) Furnishing the marketing effort sometimes decreases the profits of both suppliers, and the supplier under the decentralized structure may not provide the marketing effort if the competitor uses the direct-sales channel structure. (ii) Suppliers may benefit from the distribution channel structure that mitigates retail competitiveness, which is consistent with the results in McGuire and Staelin (1983) and Atkins and Liang (2010). However, we find some completely different results when considering the customer's marketing effort sensitivity level. (iii) Different with Cachon and Harker (2002), we find that it is impossible that the supplier benefits from a unilateral motivation to adopt the distribution mode when the marketing effort is considered. Finally, from the perspective of equilibrium on channel structure strategies, we believe that there is always an equilibrium channel structure that neither of suppliers adopts the distribution strategy. Also, there will be another equilibrium channel structure that both suppliers choose to adopt the distribution strategy when retail competition is very intense.

The remainder of the paper is organized as follows. Section 2 gives the model description and basic assumption. Section 3 explores a competition model with one of two suppliers providing marketing effort while Section 4 considers a competition model with both suppliers providing marketing effort. Section 5 reports motivations for marketing effort and channel structure selection. We summarize the results and point out directions for future research in Section 6.

\section{Model Description and Basic Assumption}

\subsection{Problem Description}

We assume there are two suppliers (denoted as $S_{i}$, i=1,2) competing for market demand. When faced with a competitor that provides a marketing effort, the supplier has to decide on channel structure and marketing effort strategies to guarantee the maximization of her profits. We assume that both manufacturers have the same unit production cost denoted as c, and the retail price of products provided by $S_{i}$ is denoted as $p_{i}$. Each supplier has two channel structure options for selling her products. When the supplier adopts a direct sales strategy, it is considered centralized. When the supplier adopts a distribution channel strategy, it means that the supplier sells through a private 
retailer and charges the retailer a wholesale price per unit of the product (denoted as $w_{i}$ ). According to the channel structure strategy adopted by the supplier and a competitor, we consider these four combinations of channel structures: (i) channel structure CC (neither the supplier nor the competitor adopts the distribution mode, hence both supply chains are centralized), (ii) channel structure CD or DC (only one of the supplier or the competitor adopts the distribution mode), and (iii) channel structure DD (both the supplier and the competitor adopt the distribution mode, hence both supply chains are decentralized). CC and DD are called pure channel structures. CD and DC are called hybrid channel structures in our paper.

Our work mainly focuses on the following three aspects: (i) the supplier's optimal solutions on pricing, order quantity, marketing effort level and profit for different channel structure and marketing effort strategies; (ii) the supplier's strategic motivations for marketing effort and channel structure selection; (iii) the strategic effects of the supplier's motivations on the competitor's profits. To simplify the description, hereinafter, we describe the two suppliers as "the supplier" $\left(S_{1}\right)$ and "the competitor" $\left(S_{2}\right)$. For convenience, in our paper we refer to a supplier's strategic motivation as the incentive to choose a strategy based on expected profits. Meanwhile, strategic effect refers to the competitor's response to any of the supplier's decisions. This strategic effect focuses on a dynamic change based on the interplay between two competing suppliers.

\subsection{Basic Assumptions}

Before establishing the basic demand functions, some assumptions should be presented.

Assumption 1. Price and marketing effort level are the key factors affecting the demand. The demand is a linear function of the sales price and an exponential function of the marketing effort level (Ghosh and Shah 2012).

Assumption 2. The costs of the product consist of fixed costs and additional innovation or setup costs depending on whether the supplier makes marketing effort. The additional costs are assumed to be a quadratic function of the marketing effort level. That is to say, the marketing effort (or investment) level of $S_{i}$, denoted as $e_{i}$, will lead to an additional quantity $\left(\beta \sqrt{e_{i}}\right)$ in market demand, where Parameter $\beta$ measures the demand sensitivity to the marketing effort level and $0 \leq \beta \leq 1$. This is similar to the demand assumptions used in existing literature (Chen and Xiao 2009, Desiraju and Moorthy 1997).

Therefore, according to Assumptions 1 and 2, the marketing effort-dependent demand function is given as follows:

$$
q_{i}=a-p_{i}+\gamma p_{j}+\beta \sqrt{e_{i}}, i, j=1,2 \text {, and } i \neq j .
$$

As a remark, when $\beta=0$, Eq. (1) can be retrogressive to the traditional Bertrand retail competition model (Atkins and Liang 2010). Parameter a represents the market potential and $\gamma(0 \leq$ $\gamma \leq 1)$ measures the degree of differentiation or the coefficient of the retail competition between two substitutable products. Parameter $e_{i}$ reflects the marketing effort level of $S_{i}$. The market demand for each supplier depends on the retail prices charged for both types of products and the marketing effort made by the suppliers. More precisely, the demand function implies two key regularities based on empirical studies (Zhu and He 2016): (i) An increase in retail price of the product from $S_{1}$ will lead to a decrease in demand for the product and a simultaneous increase in demand for the product from $S_{2}$; (ii) an increase in marketing effort can lead to an increase in demand.

Assumption 3. In order to obtain a non-negative profit, the following conditions must be satisfied: $a>c$ and $c \leq w_{i} \leq p_{i}(i=1,2)$.

Because of the different marketing effort strategies adopted by the two competing suppliers, comparisons under asymmetric competitions can provide us with additional insight, as follows in Section 3.

\section{Model I: One of Two Suppliers Provides Marketing Effort}

In this section, we consider two competitive supply chains where one supplier provides marketing effort and the other does not. According to Eq. (1), the demands from both suppliers can be written as $q_{1}=a-p_{1}+\gamma p_{2}$ and $q_{2}=a-p_{2}+\gamma p_{1}+\beta \sqrt{e_{2}}$, respectively. For Model I, we consider the following four channel structures depending on which 
distribution strategy the suppliers adopt. Obtaining these results is exceptionally tedious and adds little value. For the reader's convenience, we place the details of the derivations in the Appendix.

Scenario 1: Channel structure CC

For the CC structure, both suppliers sell their products directly to consumers, and we can regard both of them as adopting a direct-sales strategy. Both suppliers' equilibrium decisions and profits can be obtained from the following:

$$
\left\{\begin{array}{l}
\max \Pi_{S 1}^{C C}\left(p_{1} \mid p_{2}\right)=\left(p_{1}-c\right) q_{1} \\
\max \Pi_{S 2}^{C C}\left(p_{2}, e_{2} \mid p_{1}\right)=\left(p_{2}-c\right) q_{2}-e_{2} .
\end{array}\right.
$$

\section{Scenario 2: Channel structure CD}

For channel structure CD, $S_{1}$ sells directly to consumers, while $S_{2}$ sells through a retailer $\left(R_{2}\right)$. Given the marketing effort from $S_{2}$, we divide the game sequence into two steps as follows:

(i) $S_{2}$ decides on a marketing effort level and sells to downstream retailer $R_{2}$ at the unit wholesale price;

(ii) $R_{2}$ and $S_{1}$ engage in retail competition by simultaneously and independently determining their respective retail prices.

All parties' equilibrium decisions and profits can be obtained from the following two-level programming:

$$
\left\{\begin{array}{l}
\max \Pi_{S 2}^{C D}\left(w_{2}, e_{2}\right)=\left(w_{2}-c\right) q_{2}-e_{2} \\
\text { s.t., }\left\{\begin{array}{l}
\max \Pi_{R 2}^{C D}\left(p_{2} \mid p_{1}, w_{2}\right)=\left(p_{2}-w_{2}\right) q_{2} \\
\max \Pi_{S 1}^{C D}\left(p_{1} \mid p_{2}\right)=\left(p_{1}-c\right) q_{1} .
\end{array}\right.
\end{array}\right.
$$

Scenario 3: Channel structure DC

For channel structure DC, $S_{1}$ sells through a retailer $\left(R_{1}\right)$, while $S_{2}$ sells directly to consumers. The game sequence is as follows.

(i) $S_{1}$ sells to her downstream retailer $R_{1}$ at the unit wholesale price;

(ii) $R_{1}$ and $S_{2}$ engage in retail competition by simultaneously and independently determining their respective retail prices. $S_{2}$ also decides on her marketing effort level.

The equilibrium decisions and profits can be obtained from the following two-level programming:

$$
\left\{\begin{array}{l}
\max \Pi_{S 1}^{D C}\left(w_{1} \mid p_{1}, p_{2}\right)=\left(w_{1}-c\right) q_{1} \\
\text { s.t., }\left\{\begin{array}{l}
\max \Pi_{R 1}^{D C}\left(p_{1} \mid p_{2}, w_{1}\right)=\left(p_{1}-w_{1}\right) q_{1} \\
\max \Pi_{S 2}^{D C}\left(p_{2}, e_{2} \mid p_{1}\right)=\left(p_{2}-c\right) q_{2}-e_{2} .
\end{array}\right.
\end{array}\right.
$$

Scenario 4: Channel structure DD

For channel structure DD, both suppliers employ the distribution strategy to sell their products through downstream retailers. The game sequence is divided into two steps as follows.

(i) Both suppliers propose the unit wholesale prices to their respective retailers, and $S_{2}$ needs to decide on her marketing effort level;

(ii) Both retailers engage in retail competition by simultaneously and independently determining their respective retail prices.

Both suppliers' optimal decisions can be obtained from the following two-level programming:

$$
\left\{\begin{array}{l}
\left\{\begin{array}{l}
\max \Pi_{S 1}^{D D}\left(w_{1}\right)=\left(w_{1}-c\right) q_{1} \\
\max \Pi_{S 2}^{D D}\left(w_{2}, e_{2}\right)=\left(w_{2}-c\right) q_{2}-e_{2}
\end{array}\right. \\
\text { s.t., }\left\{\begin{array}{l}
\max \Pi_{R 1}^{D D}\left(p_{1} \mid p_{2}, w_{1}\right)=\left(p_{1}-w_{1}\right) q_{1} \\
\max \Pi_{R 2}^{D D}\left(p_{2} \mid p_{1}, w_{2}\right)=\left(p_{2}-w_{2}\right) q_{2} .
\end{array}\right.
\end{array}\right.
$$

The optimal equilibrium decisions and profits for the above four scenarios are given in the following theorem:

Theorem 1 The equilibrium solutions for the four different channel structures are presented in Table 1.

First, for each channel structure, we derive the underlying relationships between the two competing suppliers in terms of the equilibrium decisions and profits, which are presented in the following corollary:

Corollary 1 The equilibrium decisions and profits have the following relationships.

(i) Pure channel structures:

$\left\{\begin{array}{l}p_{1}^{* C C} \leq p_{2}^{* C C}, q_{1}^{* C C} \leq q_{2}^{* C C} \\ \Pi_{S 1}^{* C C} \leq \Pi_{S 2}^{* C C}\end{array}\right.$ and $\left\{\begin{array}{l}p_{1}^{* D D} \leq p_{2}^{* D D}, q_{1}^{* D D} \leq q_{2}^{* D D}, w_{1}^{* D D} \leq w_{2}^{* D D} \\ \Pi_{S 1}^{* D D} \leq \Pi_{S 2}^{* D D}\end{array}\right.$, where the equalities hold if and only if $\beta=\gamma=0$;

(ii) Hybrid channel structures: 


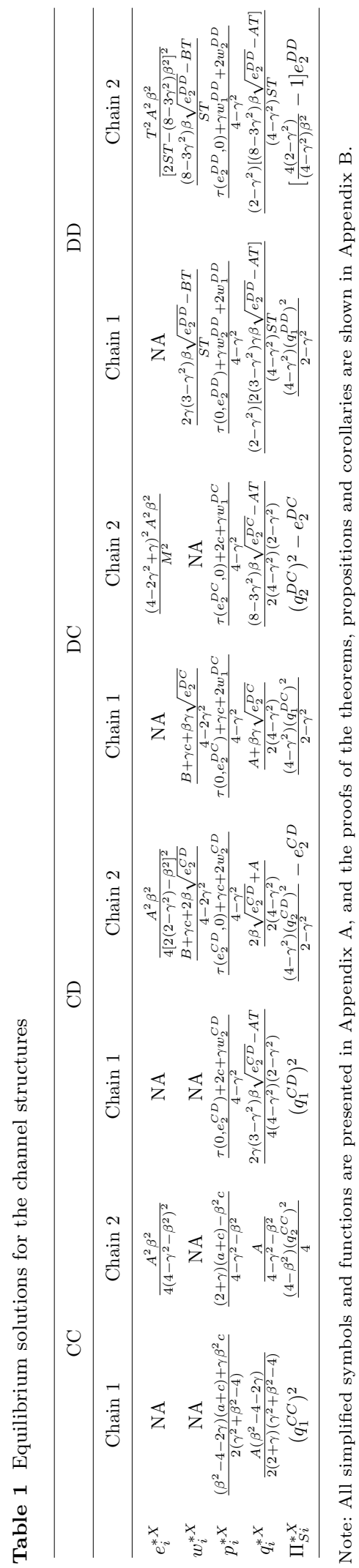

$\left\{\begin{array}{l}p_{1}^{* C D}<p_{2}^{* C D}, q_{1}^{* C D}>q_{2}^{* C D} \text { and } \\ \Pi_{S 1}^{* C D} \geq \Pi_{S 2}^{* C D}\end{array}\right.$
$\left\{\begin{array}{l}p_{1}^{* D C}>p_{2}^{* D C} \\ \Pi_{S 1}^{* D C} \leq \Pi_{S 2}^{* D C}\end{array}, q_{1}^{* D C}<q_{2}^{* D C}\right.$

From Part (i) of Corollary 1, we find that under the pure channel structures when both suppliers employ the same channel structure option, both retail price and sales quantity for $S_{2}$ are higher than those from $S_{1}$. On the one hand, compared with the product from $S_{1}$, the total costs of the product from $S_{2}$ are higher due to the investment in marketing effort. The mark-up will inevitably lead to an increase in retail price or unit wholesale price. On the other hand, the marketing effort made by $S_{2}$ increases the demand for the product from $S_{2}$, even at a higher retail price. Therefore, we believe that when both suppliers employ the same channel structure, the supplier who makes marketing effort gains more profits than those without making marketing effort.

Second, under the hybrid channel structures when both suppliers employ different channel structure options, Part (ii) offers different insight. Both suppliers' decisions and their profits depend on the channel structures. Specifically, the directsales supplier has a lower retail price and a higher order quantity relative to the distribution channel supplier. Meanwhile, under the hybrid channel structures, the direct-sales supplier always benefits more than the distribution channel supplier regardless of whether the marketing effort is made. Thus, at this point the impact of channel structure decisions on suppliers' profitability prevails over that of marketing effort strategies.

The following proposition provides comparisons of profits under different equilibrium channel structures and reveals insight into strategies for selecting a channel structure.

Proposition 1 From the perspective of channel structure strategy, we have

(i) $\forall \gamma, \beta,\left\{\begin{array}{l}\Pi_{S 1}^{* C C}>\Pi_{S 1}^{* D C} \\ \Pi_{S 2}^{* C C}>\Pi_{S 2}^{* C D}\end{array}\right.$;

(ii) If $(\gamma, \beta) \in\left\{\gamma_{1} \leq \gamma \leq \gamma_{2}, \beta_{1}<\beta \leq 1\right\} \cup\left\{\gamma_{2} \leq\right.$ $\gamma \leq 1,0 \leq \beta \leq 1\}, \Pi_{S 1}^{* D D}>\Pi_{S 1}^{* C D}$; otherwise, $\Pi_{S 1}^{* D} D \leq \Pi_{S 1}^{* C D}$;

(iii) If $(\gamma, \beta) \in\left\{\gamma_{3} \leq \gamma \leq \gamma_{4}, \beta_{2}<\beta \leq 1\right\} \cup\left\{\gamma_{4}<\right.$ $\gamma \leq 1,0 \leq \beta \leq 1\}, \Pi_{S 2}^{* D D}>\Pi_{S 2}^{* D \bar{C}}$; otherwise, $\Pi_{S 2}^{* \bar{D} D} \leq \Pi_{S 2}^{* D C}$, where the thresholds $\gamma_{1}, \gamma_{2}, \beta_{1}, \gamma_{3}$, 
$\gamma_{4}$ and $\beta_{2}$ are uniquely determined by the equations as $Z_{1}\left(0 \mid \gamma_{1}\right)=0, Z_{1}\left(1 \mid \gamma_{2}\right)=0, Z_{1}\left(\beta_{1} \mid \gamma \in\right.$ $\left.\left[\gamma_{1}, \gamma_{2}\right]\right)=0, Z_{2}\left(1 \mid \gamma_{3}\right)=0, Z_{2}\left(0 \mid \gamma_{4}\right)=0$ and $Z_{2}\left(\beta_{2} \mid \gamma \in\left[\gamma_{3}, \gamma_{4}\right]\right)=0$, respectively.

Proposition 1 shows how $S_{1}$ 's channel structure strategy affects equilibrium profits and suggests the following insight: (i) When one of the two suppliers (regardless of whether she makes marketing effort) sells directly to consumers, the other will adopt the direct sales strategy as well. Moreover, when both suppliers take the same channel strategy, $S_{2}$ who makes marketing effort always gains more profits than $S_{1}$ (i.e., $\Pi_{S 2}^{* C C} \geq \Pi_{S 1}^{* C C}$ and $\Pi_{S 2}^{* D D} \geq \Pi_{S 1}^{* D D}$ ). (ii) If one of the suppliers sells through a retailer, the other will prefer to choose a direct-sales strategy when the demand sensitivity to marketing effort is sufficiently low and the market is not intense. i.e., $\Pi_{S 1}^{* D D} \leq \Pi_{S 1}^{* C D}$ for $\beta<\beta_{1}$ and $\gamma<\gamma_{1}\left(\Pi_{S 2}^{* D D} \leq \Pi_{S 2}^{* D C}\right.$ for $\beta<\beta_{2}$ and $\gamma<\gamma_{3}$ ). Otherwise, the supplier benefits more from the distribution channel option.

According to Proposition 1, there are two equilibria on the channel structure decisions. First, channel structure $\mathrm{CC}$ is an equilibrium structure over a defined area. That is, the supplier's profit under a direct-sales strategy is always more than the profit that each supplier could realize by exercising the outside option. Second, we find that when retail competition is very intense and the demand sensitivity to marketing effort is relatively high (i.e., $\gamma \in\left[\gamma_{3}, \gamma_{4}\right]$ for $\forall \beta \in\left(\beta_{2}, 1\right]$ and $\gamma \in\left(\gamma_{4}, 1\right]$ for $\left.\forall \beta \in[0,1]\right)$, channel structure DD becomes an equilibrium structure under these conditions: $\Pi_{S 1}^{* D D}>\Pi_{S 1}^{* C D}$ and $\Pi_{S 2}^{* D D}>\Pi_{S 2}^{* D C}$. Note that if $\gamma>\gamma_{4}$, the conditions $\gamma_{2}<\gamma_{4}$ and $\gamma>\gamma_{2}$ can always be satisfied. Finally, we find that neither channel structure CD nor channel structure DC is an equilibrium structure based on the similar game approach. It means that no player can "do it alone" (unilaterally change the channel structure decision) to increase profits.

The following Figure 1 shows the regions for both suppliers' equilibrium channel structure strategies. In the left area of the curve $\Pi_{S 2}^{* D D}=$ $\Pi_{S 2}^{* D C}$, channel structure $\mathrm{CC}$ is the only equilibrium channel structure (ECS). In the right area of the curve, channel structures CC and DD are both equilibrium channel structures (ECS).

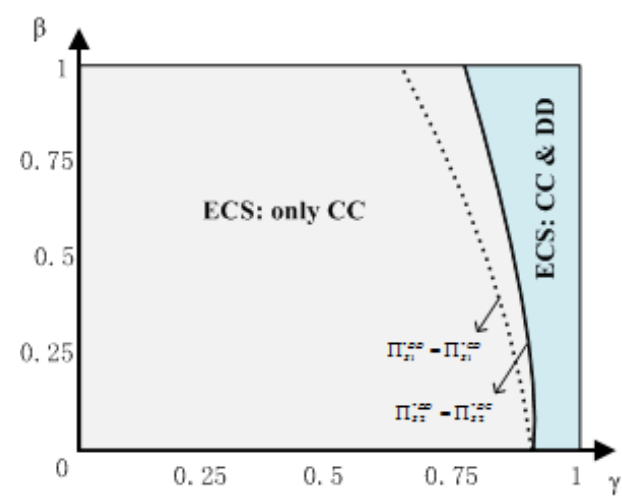

Fig. 1 Equilibrium channel structure in Model I

Interestingly, from the perspective of equilibrium, retail competition reveals itself in the pure channel structure, i.e., the direct competition between two suppliers or between downstream retailers is very common in reality, but there is no direct competition in any hybrid channel structures (i.e., no competition between one supplier and the other supplier's downstream retailer). It implies that for the supplier, competing with a player at the same level is a wise strategy in terms of its own interest. In the following, we will focus on the effects of the marketing effort sensitivity coefficient $(\beta)$ on equilibrium profits under channel structures CC and DD. Our findings are presented as follows:

Corollary 2 The relationships between the $S_{i}$ 's profit and the customer's sensitivity to marketing effort are as follows, where $i=1,2$.

(i) For the $C C$ structure, $\partial \Pi_{S i}^{* C C} / \partial \beta \geq 0, \forall \gamma, \beta$; (ii) For the $D D$ structure, $S_{1}$ 's profit satisfies $\partial \Pi_{S 1}^{* D D} / \partial \beta \geq 0, \forall \gamma, \beta ;$ and $S_{2}$ 's profit satisfies $\begin{cases}\partial \Pi_{S 2}^{* D D} / \partial \beta \leq 0 & (\gamma, \beta) \in\left\{0 \leq \gamma \leq \gamma_{5}, \beta_{3}<\beta \leq 1\right\} \\ \partial \Pi_{S 2}^{* D} D / \partial \beta \geq 0 & \text { otherwise }\end{cases}$ where the thresholds $\gamma_{5}$ and $\beta_{3}$ are uniquely determined by the equations as $Z_{3}\left(1 \mid \gamma_{5}\right)=0$ and $Z_{3}\left(\beta_{3} \mid \gamma\right)=0$, respectively.

For the equilibrium channel structure CC, an increase in the marketing effort sensitivity coefficient results in increases of both suppliers' profits. On the one hand, as the market becomes more sensitive to marketing effort provided by $S_{2}$, the demand from $S_{2}$ will increase, which will naturally lead to an increase in $S_{2}$ 's profit. On the other hand, $S_{1}$ will also benefit from the increased 
demand from $S_{2}$. Table 1 shows that with the increase of the marketing effort sensitivity coefficient, the product demand from $S_{1}$ also increases. For the equilibrium channel structure DD, an increase in the sensitivity coefficient improves $S_{1}$ 's profits but does not always improve $S_{2}$ 's profit. For example, from Part (ii) of Corollary 2, we find that when the sensitivity coefficient is sufficiently high and the market intensity $(\gamma)$ is sufficiently low, $S_{2}$ 's profit will decrease with the marketing effort sensitivity coefficient. The reason behind this is that the relatively high marketing effort sensitivity directly leads to more market demand from $S_{2}$. However, under a more moderate market, $S_{2}$ chooses to sell through a retailer. $S_{2}$ loses the opportunity to properly price her product. Ultimately, an increase in the sensitivity coefficient will result in a decrease of $S_{2}$ 's profit. By contrast, if the sensitivity coefficient is sufficiently low or the market intensity is sufficiently high, $S_{2}$ 's profits will increase with an increase in the marketing effort sensitivity coefficient. Therefore, under a non-intense market, it is injudicious for a supplier with a distribution channel strategy to blindly increase her investment on marketing effort.

\section{Model II: Both of Suppliers Provide Marketing Effort}

In this section, we consider two competitive supply chains where both suppliers provide the marketing effort for product sales. The market demand for $S_{i}$ is $\tilde{q}_{i}=a-\tilde{p}_{i}+\gamma \tilde{p}_{j}+\beta \sqrt{\tilde{e}_{i}},(i, j=1,2$ and $i \neq j$ ). Due to the symmetry of the two channels, the following three scenarios should be studied.

Scenario 1: Channel structure CC

Under channel structure CC, both suppliers sell their products directly to consumers. The equilibrium decisions and profits can be obtained from the following Nash game between two suppliers.

$$
\left\{\begin{array}{l}
\max \tilde{\Pi}_{S 1}^{C C}\left(\tilde{p}_{1}, \tilde{e}_{1}\right)=\left(\tilde{p}_{1}-c\right) \tilde{q}_{1}-\tilde{e}_{1} \\
\max \tilde{\Pi}_{S 2}^{C C}\left(\tilde{p}_{2}, \tilde{e}_{2}\right)=\left(\tilde{p}_{2}-c\right) \tilde{q}_{2}-\tilde{e}_{2}
\end{array}\right.
$$

Scenario 2: Channel structure CD or DC

Under channel structure CD, $S_{1}$ sells directly to consumers, and $S_{2}$ sells through a downstream retailer. Similar to Model I, equilibrium decisions and profits can be obtained by solving the following two-level programming:

$$
\left\{\begin{array}{c}
\max \tilde{\Pi}_{S 2}^{C D}\left(\tilde{w}_{2}, \tilde{e}_{2}\right)=\left(\tilde{w}_{2}-c\right) \tilde{q}_{2}-\tilde{e}_{2} \\
\text { s.t., }\left\{\begin{array}{l}
\max \tilde{\Pi}_{R 2}^{C D}\left(\tilde{p}_{2} \mid \tilde{p}_{1}, \tilde{w}_{2}\right)=\left(\tilde{p}_{2}-\tilde{w}_{2}\right) \tilde{q}_{2} \\
\max \tilde{\Pi}_{S 1}^{C D}\left(\tilde{p}_{1}, \tilde{e}_{1} \mid \tilde{p}_{2}, \tilde{w}_{2}\right)=\left(\tilde{p}_{1}-c\right) \tilde{q}_{1} \\
-\tilde{e}_{1} .
\end{array}\right.
\end{array}\right.
$$

Due to this symmetry, the equilibrium decisions for the DC structure can be obtained similarly.

Scenario 3: Channel structure DD

Under channel structure DD, both suppliers adopt the distribution strategy. Similar to Model I, equilibrium decisions and profits can be obtained by solving the following two-level programming.

$$
\left\{\begin{array}{l}
\max \tilde{\Pi}_{S i}^{D D}\left(\tilde{w}_{i}, \tilde{e}_{i}\right)=\left(\tilde{w}_{i}-c\right) \tilde{q}_{i}-\tilde{e}_{i}, \quad i=1,2 \\
\text { s.t., }\left\{\begin{array}{l}
\max \tilde{\Pi}_{R 1}^{D D}\left(\tilde{p}_{1} \mid \tilde{p}_{2}, \tilde{w}_{1}\right)=\left(\tilde{p}_{1}-\tilde{w}_{1}\right) \tilde{q}_{1} \\
\max \tilde{\Pi}_{R 2}^{D D}\left(\tilde{p}_{2} \mid \tilde{p}_{1}, \tilde{w}_{2}\right)=\left(\tilde{p}_{2}-\tilde{w}_{2}\right) \tilde{q}_{2} .
\end{array}\right.
\end{array}\right.
$$

All equilibrium decisions for the four channel structures are summarized in the following theorem:

Theorem 2 The equilibrium solutions for the four channel structures are presented in Table 2.

According to Theorem 2, we first compare the equilibrium decisions and profits between two competing suppliers for each channel structure. Obviously, due to symmetry, for each pure channel structures (CC or DD), the corresponding decisions and profits of $S_{i}$ equal those of $S_{j}$, e.g., $\tilde{p}_{1}^{* C C}=\tilde{p}_{2}^{* C C}, \tilde{p}_{1}^{* D D}=\tilde{p}_{2}^{* D D}, \tilde{\Pi}_{S 1}^{* C C}=\tilde{\Pi}_{S 2}^{* C C}$ and $\tilde{\Pi}_{S 1}^{* D D}=\tilde{\Pi}_{S 2}^{* D D}$. For the hybrid CD or DC structure, we have the following results:

Corollary 3 Comparing $C D$ and $D C$, we have

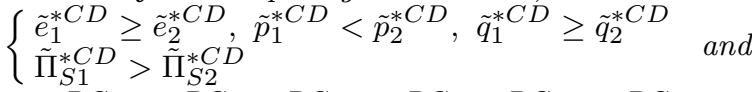
$\left\{\begin{array}{l}\tilde{e}_{1}^{* D C} \leq \tilde{e}_{2}^{* D C}, \tilde{p}_{1}^{* D C}>\tilde{p}_{2}^{* D C}, \tilde{q}_{1}^{* D C} \leq \tilde{q}_{2}^{* D C} \\ \tilde{\Pi}_{S 1}^{* D C}<\tilde{\Pi}_{S 2}^{* D C}\end{array}\right.$.

From Corollary 3, we find that in the CD structure, a supplier who sells directly to consumers provides a higher level of marketing effort, a lower retail price and a higher order quantity than the 
Table 2 Equilibrium solutions for the different channel structures, where i=1, 2 .

\begin{tabular}{|c|c|c|c|c|}
\hline & $\mathrm{CC}$ & \multicolumn{2}{|c|}{$\mathrm{CD}(\mathrm{DC})$} & $\mathrm{DD}$ \\
\hline$\tilde{e}_{i}^{* X}$ & $\begin{array}{c}\text { Chain i } \\
\frac{(a-c+\gamma c)^{2} \beta^{2}}{\left(4-\beta^{2}-2 \gamma\right)^{2}}\end{array}$ & $\begin{array}{l}\text { Chain 1(2) } \\
{\left[\frac{A \varphi \beta}{2 M-\phi \beta^{2}}\right]^{2}}\end{array}$ & $\begin{array}{l}\text { Chain 2(1) } \\
{\left[\frac{A N \beta}{2 M-\phi \beta^{2}}\right]^{2}}\end{array}$ & {$\left[\frac{\beta A}{2 S+(\gamma+2) \beta^{2}}\right]^{2}$} \\
\hline$\tilde{w}_{i}^{* X}$ & NA & NA & $\frac{\left(2-\gamma^{2}+\gamma\right) c+\tau\left(\tilde{e}_{2}^{C D}, \tilde{e}_{1}^{C D}\right)}{4-2 \gamma^{2}}$ & $\frac{T \varphi+\left(2 \gamma^{3}-6 \gamma+3 \gamma^{2}-8\right) \beta \sqrt{\tilde{e}_{i}^{D D}}}{\gamma^{2}-4\left(\gamma^{2}-2\right)^{2}}$ \\
\hline$\tilde{p}_{i}^{* X}$ & $\frac{2 a+2 c-\beta^{2} c}{4-\beta^{2}-2 \gamma}$ & $\frac{2 c+\tau\left(\tilde{e}_{1}^{C D}, \tilde{e}_{2}^{C D}\right)+\gamma \tilde{w}_{2}^{C D}}{4-\gamma^{2}}$ & $\frac{\gamma c+\tau\left(\tilde{e}_{2}^{C D}, \tilde{e}_{1}^{C D}\right)+2 \tilde{w}_{2}^{C D}}{4-\gamma^{2}}$ & $\frac{a+\beta \sqrt{\tilde{e}_{i}^{D D}}+\tilde{w}_{i}^{D D}}{2-\gamma}$ \\
\hline $\begin{array}{l}\tilde{q}_{i}^{* X} \\
\tilde{\Pi}_{S i}^{* X}\end{array}$ & $\begin{array}{l}\frac{2(a-c+\gamma c)}{4-\beta^{2}-2 \gamma} \\
\frac{\left(4-\beta^{2}\right) \tilde{e}_{i}^{C C}}{\beta^{2}}\end{array}$ & $\begin{array}{c}\frac{2 \sqrt{\tilde{e}_{1}^{C D}}}{\beta} \\
\frac{\left(4-\beta^{2}\right) \tilde{e}_{1}^{C D}}{\beta^{2}}\end{array}$ & $\begin{array}{c}\frac{\left(\gamma^{2}+\gamma-2\right) c+\tau\left(\tilde{e}_{2}^{C D}, \tilde{e}_{1}^{C D}\right)}{2\left(4-\gamma^{2}\right)} \\
{\left[\frac{4\left(2-\gamma^{2}\right)}{\left(4-\gamma^{2}\right) \beta^{2}}-1\right] \tilde{e}_{2}^{C D}}\end{array}$ & $\frac{\frac{a+\beta \sqrt{\tilde{e}_{i}^{D D}}+(\gamma-1) \tilde{w}_{i}^{D D}}{2-\gamma}}{\left[\frac{4\left(2-\gamma^{2}\right)}{\left(4-\gamma^{2}\right) \beta^{2}}-1\right] \tilde{e}_{i}^{D D}}$ \\
\hline
\end{tabular}

supplier who sells through a retailer. A supplier who uses a direct sales strategy benefits from her marketing effort more effectively than a supplier who uses a distribution channel strategy. It avoids the weakening of the marketing effort-dependent advantage by the middleman. In addition, the products sold with a direct-sales strategy will gain more market shares and profits than those sold with a distribution strategy due to the preferential retail price. Noticeably, a pull inventory configuration from the direct-sales decision (centralized supply chain structure) generally outperforms a push configuration from the distribution decision (decentralized supply chain structure).

It is well known that channel conflict exists between the distribution channel (i.e., traditional brick and mortar stores) and the direct-sales channel (i.e., online stores) because the marketing effort is provided in both channels. The discussion above shows that the supplier can benefit more from adopting a direct-sales strategy. To avoid the channel conflict, Xiao et al. (2014) design a dual channel strategy and make a careful trade-off between product variety and customization and their research cites Nike as a successful example. One of the advantages of distribution channel strategy, however, is that the downstream retailer has the opportunity to invest in store assistance efforts to reduce product returns (Xia et al. 2016). In this case, the supplier who sells through a retailer may be more profitable than those who sell directly to consumers.

Next, we explore the impacts of the channel structure strategy on the supplier's profit by comparing the profits of a supplier and her competitor with different channel structure options. The following proposition gives the results from the profit comparison under the different channel structures.

Proposition 2 When both suppliers make marketing effort, we have

(i) $\forall \gamma, \beta,\left\{\begin{array}{l}\tilde{\Pi}_{S 1}^{* C C}>\tilde{\Pi}_{S 1}^{* D C} \\ \tilde{\Pi}_{S 2}^{* C C} \geq \tilde{\Pi}_{S 2}^{* C D}\end{array} ;\right.$

(ii) If $(\gamma, \beta) \in\left\{\gamma_{6}<\gamma \leq \gamma_{7}, \beta_{4}<\beta \leq 1\right\} \cup\left\{\gamma_{7}<\right.$ $\left.\gamma<\gamma_{8}, 0 \leq \beta \leq 1\right\} \cup\left\{\gamma_{8}<\gamma<1,0 \leq \beta \leq \beta_{5}\right\}$, $\left\{\begin{array}{l}\tilde{\Pi}_{S 1}^{* D D} \geq \tilde{\Pi}_{S 1}^{* C D} \\ \tilde{\Pi}_{S 2}^{* D D} \geq \tilde{\Pi}_{S 2}^{* D C}\end{array}\right.$, where the thresholds $\gamma_{6}, \gamma_{8}, \gamma_{7}, \beta_{4}$ and $\beta_{5}$ are uniquely determined by $Z_{4,5}\left(1 \mid \gamma_{6}, \gamma_{8}\right)=$ $0, Z_{4,5}\left(0 \mid \gamma_{7}\right)=0, Z_{4,5}\left(\beta_{4} \mid \gamma \in\left[\gamma_{6}, \gamma_{7}\right]\right)=0$ and $Z_{4,5}\left(\beta_{5} \mid \gamma \in\left[\gamma_{8}, 1\right]\right)=0$, respectively.

Proposition 2 implies $S_{1}$ 's optimal channel structure strategy. Specifically, according to Part (i), when the competitor $\left(S_{2}\right)$ sells directly to consumers, $S_{1}$ will obtain more profits from the direct-sales option (centralized decision) than from the distribution option (decentralized decision). In other words, if the competitor $\left(S_{2}\right)$ sells directly to consumers, it is optimal for $S_{1}$ to follow suit. Obviously, channel structure CC is an equilibrium channel structure (ECS) for both suppliers. From Part (ii), provided that some conditions are satisfied, i.e., the market intensity is relatively high and the customer's sensitivity to the supplier's effort performance is relatively low, we believe that DD structure is also an equilibrium channel structure. When the competitor $\left(S_{2}\right)$ distributes products, $S_{1}$ obtains higher profits from the decentralized decision than the centralized decision. Therefore, there are two equilibrium 
channel structures (ECS): the CC and DD channel structures. Figure 2 illustrates the equilibrium in Model II.

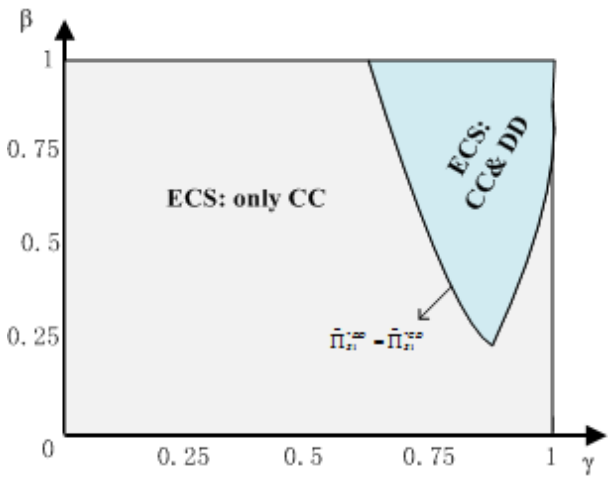

Fig. 2 Equilibrium channel structure in Model II

For the above two equilibrium structures CC and DD, the following corollary studies the effects of the marketing effort sensitivity level $(\beta)$ on both suppliers' equilibrium profits.

Corollary 4 The relationships between $S_{i}$ 's profit and the marketing effort sensitivity level are as follows, where $i=1$, 2 .

(i) Under channel structure $C C, \forall \gamma, \beta, \partial \tilde{\Pi}_{S i}^{* C C} / \partial \beta \geq$ 0 ;

(ii) Under channel structure $D D$, if $(\gamma, \beta) \in\{0 \leq$ $\left.\gamma \leq \gamma_{9}, \beta_{6}<\beta \leq 1\right\} \cup\left\{\gamma_{10} \leq \gamma \leq 1, \beta_{6}<\beta \leq 1\right\}$, $\partial \tilde{\Pi}_{S i}^{* D D} / \partial \beta \leq 0$; otherwise, $\partial \tilde{\Pi}_{S i}^{* D D} / \partial \beta \geq 0$, where the thresholds $\gamma_{9}, \gamma_{10}$ and $\beta_{6}$ are uniquely determined by $Z_{6}\left(1 \mid \gamma_{9}, \gamma_{10}\right)=0$ and $Z_{6}\left(\beta_{6} \mid \gamma\right)=0$, respectively.

From Corollary 4, we find that in the CC structure, an increase in marketing effort sensitivity level leads to an increase in profits for both suppliers. In other words, the marketing effort sensitivity level of the supplier who sells directly to consumers enhances her profitability. However, in the DD structure, the profits of both suppliers decrease with the marketing effort sensitivity level, when the marketing effort sensitivity level is sufficiently high (i.e., $\beta_{6}<\beta \leq 1$ ) and the retail competition is either very low or very intense (i.e., $0 \leq \gamma \leq \gamma_{9}$ or $\left.\gamma_{10} \leq \gamma \leq 1\right)$. On the one hand, a very low competition weakens the advantage of the suppliers' distribution strategy. The double marginalization effect may result in a decline in profits for both suppliers. At this point, the raised retail price resulting from the indirect sales strategy will in turn lead to a decrease in order quantity. Thus, as the sensitivity increases, the profitability of supplier decreases. On the other hand, a very intense market implies a surge in demand, because of the relatively high marketing effort sensitivity. Simultaneously, the very intense competition between both retailers likely results in a substantial decline in prices. As a result, the suppliers will have a decrease in profit with the marketing effort sensitivity level. Moreover, from a perspective of the DD channel structure, when the marketing effort sensitivity of consumers is relatively low (i.e., $\beta<\beta_{6}$ ), the impact of the retail price on profits tends to be greater than that of marketing effort.

\section{Motivations for Marketing Effort and Channel Structure Selection}

In this section, we investigate the motivations for adopting the aforementioned strategies regarding marketing effort and/or channel structure strategies when faced with a competitor that offers a substitutable product. Meanwhile, we explore the strategic effects of these motivations towards the competitor's profitability.

\subsection{Motivations for Marketing Effort}

We first investigate $S_{1}$ 's marketing effort motivations by comparing her profits with and without an effort performance plan under consideration of market competition. The main results are as follows:

Proposition 3 From the perspective of $S_{1}$, we have (i) $\left\{\begin{array}{l}\tilde{p}_{1}^{* X} \geq p_{1}^{* X}, \tilde{w}_{1}^{* D D} \geq w_{1}^{* D D} \\ \tilde{q}_{1}^{* X} \geq q_{1}^{* X},\end{array}\right.$ and $\tilde{\Pi}_{S 1}^{* X} \geq \Pi_{S 1}^{* X}$, where $X \in \Omega \backslash\{D C\}$;

(ii) $\left\{\begin{array}{l}\tilde{p}_{1}^{* D C} \geq p_{1}^{* D C}, \tilde{w}_{1}^{* D C} \geq w_{1}^{* D C} \\ \tilde{q}_{1}^{* D C} \geq q_{1}^{* D C}\end{array}\right.$ and $\left\{\begin{array}{l}\tilde{\Pi}_{S 1}^{* D C}<\Pi_{S 1}^{* D C}(\gamma, \beta) \in\left\{0 \leq \gamma \leq \gamma_{11}, \beta_{7}<\beta \leq 1\right\} \\ \tilde{\Pi}_{S 1}^{* D C} \geq \Pi_{S 1}^{* D C} \text { otherwise }\end{array}\right.$ where the thresholds $\gamma_{11}$ and $\beta_{7}$ are uniquely determined by $Z_{7}\left(1 \mid \gamma_{11}\right)=0$ and $Z_{7}\left(\beta_{7} \mid \gamma\right)=0$, respectively. Specifically, the above equalities are satisfied if and only if $\beta=0$. 
Part (i) indicates that $S_{1}$ selling directly to consumers is motivated to provide marketing effort, since the competitor $\left(S_{2}\right.$ or $\left.R_{2}\right)$ sells with a marketing effort (i.e., $\tilde{\Pi}_{S 1}^{* C D} \geq \Pi_{S 1}^{* C D}$ and $\tilde{\Pi}_{S 1}^{* C C} \geq$ $\left.\Pi_{S 1}^{* C C}\right)$. Actually, $S_{1}$ 's effort performance plan will lead to a higher unit sale price (i.e., a higher unit retail price under decentralized supply chain structure or a higher unit wholesale price under centralized supply chain structure) and a higher order quantity. Under these channel structures $X \in \Omega \backslash\{D C\}, S_{1}$ will gain more profits from making marketing effort, even when taking marketing effort costs into account. On the one hand, when both suppliers take the same channel structure option, such as CC or DD structures, $S_{1}$ increases her profits due to marketing effort stimulating the demand. On the other hand, in the CD channel structure, $S_{1}$ benefits from marketing effort as well because the competitor's distribution plan will weaken the competitiveness of the competitor due to the double marginalization.

Interestingly, when $S_{1}$ sells through a retailer, her profits may decrease under certain conditions (i.e., $0 \leq \gamma \leq \gamma_{11}$ and $\beta_{7}<\beta \leq 1$ ) provided $S_{2}$ adopts a direct-sales strategy. Obviously, when the products competition becomes less intense than the competition coefficient $\gamma_{11}$, the negative effect of double marginalization outweighs the positive effect of the marketing effort on $S_{1}$ 's profit. Therefore, we draw the conclusion that when the competitor makes marketing effort, $S_{1}$ 's decision on marketing effort level depends on the channel structure strategies of both sides as well as the competition intensity. Specifically, we give some insight as follows:

(i) $S_{1}$ is always motivated to provide the marketing effort with the direct-sales strategy, regardless of the competitor's channel structure strategy.

(ii) In spite of $S_{1}$ selling through a retailer, when the competitor adopts the distribution strategy, she intends to make marketing effort.

(iii) If the competitor uses the direct-sales strategy, we find that the distribution supplier will not provide marketing effort under the con$\operatorname{dition}(\gamma, \beta) \in\left\{0 \leq \gamma \leq \gamma_{11}, \beta_{7}<\beta \leq 1\right\}$. It implies that the effect of retail competition is more significant than that of marketing effort on $S_{1}$ 's profit. In fact, when the consumers' sensitivity to marketing effort is greater than $\beta_{7}, S_{1}$ will be more motivated to make marketing effort.
However, the distribution supplier has to refuse to provide marketing effort in order to maximize her profits $\left(\tilde{\Pi}_{S 1}^{* D C}<\Pi_{S 1}^{* D C}\right)$ when the market intensity is less than $\gamma_{11}$. This irregular operation is mainly caused by retail competition.

Now, the strategic effects of $S_{1}$ 's marketing effort on the competitor $\left(S_{2}\right)$ are considered as follows. When $S_{1}$ makes the transition from not making marketing effort to making marketing effort, it implies that the competitor will increase her marketing effort costs.

Proposition 4 The strategic effects of $S_{1}$ 's motivation for marketing effort are given as follows.

(i) The optimal decisions satisfy

$\left\{\begin{array}{l}\tilde{p}_{2}^{* X} \geq p_{2}^{* X}, \tilde{q}_{2}^{* X} \geq q_{2}^{* X}, \tilde{e}_{2}^{* X} \geq e_{2}^{* X} \\ \tilde{w}_{2}^{* C D} \geq w_{2}^{* C D}, \tilde{w}_{2}^{* D D} \geq w_{2}^{* D D}\end{array}\right.$, where $X \in \Omega ;$

(ii) The expected profits satisfy

(a) $\tilde{\Pi}_{S 2}^{* X} \geq \Pi_{S 2}^{* X}$, where $X \in \Omega \backslash\{D D\}$;

(b) If $(\gamma, \beta) \in\left\{\gamma_{12}<\gamma \leq 1, \beta_{8}<\beta \leq 1\right\}$, $\tilde{\Pi}_{S 2}^{* D D}<\Pi_{S 2}^{* D D}$; otherwise, $\tilde{\Pi}_{S 2}^{* \bar{D} D} \geq \Pi_{S 2}^{* D D}$, where $\gamma_{12}$ and $\beta_{8}$ are uniquely determined by $Z_{8}\left(1 \mid \gamma_{12}\right)=0$ and $Z_{8}\left(\beta_{8} \mid \gamma \in\left(\gamma_{12}, 1\right]\right)=0$, respectively.

Specifically, $\beta=0$ results in $\tilde{e}_{2}^{* X}=e_{2}^{* X}$ and the other equalities are satisfied if and only if either $\beta=0$ or $\gamma=0$.

From Proposition 4, when $S_{1}$ switches from not making marketing effort to making them, we find that it can stimulate the competitor's $\left(S_{2}\right.$ 's) marketing effort. $S_{2}$ 's marketing effort, order quantity, retail price or wholesale price will increase. As a matter of fact, in order to maximize profitability, both suppliers must make appropriate marketing effort, and the results reflect increases in order quantity and price. We believe that $S_{1}$ 's switch will have a positive effect on $S_{2}$ 's profits under channel structures CC, CD, and DC. However, if both suppliers sell through retailers (i.e., channel structure DD), $S_{1}$ 's marketing effort motivation can hurt $S_{2}$ 's profitability when competition intensity and the sensitivity level for marketing effort are sufficiently high (i.e., $\left.\gamma_{12}<\gamma \leq 1, \beta_{8}<\beta \leq 1\right)$. The reason may be that an increase in consumers' sensitivity to suppliers' marketing effort makes the supplier who makes marketing effort occupy a higher market share than the supplier without investments in marketing effort. In order to illustrate this intuitive conclusion, Figure 3 shows $S_{1}$ 's marketing 
effort strategies and their effects on the competitor's profit.

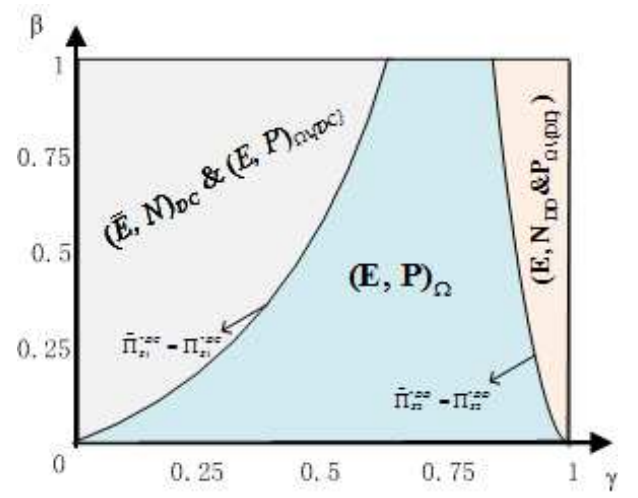

Fig. 3 Marketing effort strategy and the strategic effects

$(\bar{E}, N)_{D C}$ : Given channel structure DC, $S_{1}$ does not make marketing effort, which has a negative effect on the competitor's profit;

$(E, P)_{\Omega \backslash\{D C\}}$ : Given channel structure CC, CD or DD, $S_{1}$ makes marketing effort, which has a positive effect on the competitor's profit;

$\left(E, N_{D D} \& P_{\Omega \backslash\{D D\}}\right): S_{1}$ makes marketing effort, which has a negative effect on the competitor under channel structure $\mathrm{DD}$, and a positive effect on the competitor's profit under the other channel structures.

As Figure 3 shows, $S_{1}$ has the motivation to make marketing effort determined by $\tilde{\Pi}_{S 1}^{* D C}=$ $\Pi_{S 1}^{* D C}$ in the area below the curve. By contrast, above the curve, $S_{1}$ is not motivated to make marketing effort, especially in the DC channel structure. $S_{1}$ 's marketing effort strategy may have different effects on the competitor depending on market conditions. Intuitively, both suppliers should be better off by choosing to make marketing effort, since it stimulates market demand. We can expect that $S_{1}$ 's motivation for marketing effort will have positive effect on the competitor's profitability. However, a counterintuitive result can occur due to retail competition. When retail competition is intense, $S_{1}$ increasing its marketing effort investments can draw demand away from the competitor. Therefore, although it can increase total market demand, $S_{1}$ 's marketing effort strategy does not always have a positive impact on the competitor's profitability.

\subsection{Motivations for Channel Structure Selection}

In this section, we will study $S_{1}$ 's motivations on channel structure selection in each model by comparing $S_{1}$ 's profits under the direct-sales and the distribution strategy. Meanwhile, we will also explore the effect of $S_{1}$ 's channel structure motivation towards the competitor's decisions and profits. As summarized in Tables 1 and 2, we derive the following results:

Proposition 5 From $S_{1}$ 's perspective, we consider the following cases:

(i) Model I: (One of two suppliers makes marketing effort), we have

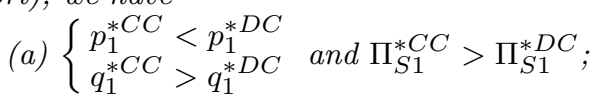
(b) $\left\{\begin{array}{l}p_{1}^{* D D} \geq p_{1}^{* C D} \\ q_{1}^{* D D} \geq q_{1}^{* C D}\end{array}\right.$ and if $(\gamma, \beta) \in\{0 \leq \gamma \leq$ $\left.\gamma_{1}, 0 \leq \beta \leq 1\right\} \cup\left\{\gamma_{1} \leq \gamma \leq \gamma_{2}, 0 \leq \beta \leq \beta_{1}\right\}, \Pi_{S 1}^{* D D} \leq$ $\Pi_{S 1}^{* C D}$; otherwise, $\Pi_{S 1}^{* \bar{D} D}>\Pi_{S 1}^{* C D}$;

(ii) Model II: (Both suppliers make marketing effort), we have

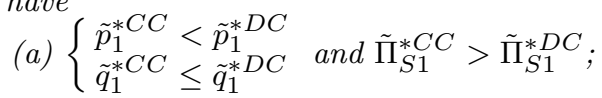

(b) $\left\{\begin{array}{l}\tilde{p}_{1}^{* D D} \geq \tilde{p}_{1}^{* C D} \\ \tilde{q}_{1}^{* D D} \geq \tilde{q}_{1}^{* C D}\end{array}\right.$ and if $(\gamma, \beta) \in\left\{\gamma_{6}<\gamma \leq\right.$ $\left.\gamma_{7}, \beta_{4}<\beta \leq 1\right\} \cup\left\{\gamma_{7}<\gamma<\gamma_{8}, 0 \leq \beta \leq\right.$ $1\} \cup\left\{\gamma_{8}<\gamma<\overline{1}, 0 \leq \beta \leq \beta_{5}\right\}, \tilde{\Pi}_{S 1}^{* D D} \geq \tilde{\Pi}_{S 1}^{* C D}$; otherwise, $\tilde{\Pi}_{S 1}^{* D D}<\tilde{\Pi}_{S 1}^{* C D}$.

Specifically, the above equalities are satisfied if and only if $\beta=0$.

Proposition 5 illustrates how under each model, $S_{1}$ 's channel structure strategy affects its quantity decisions or pricing as well as profits. First, when the competitor $\left(S_{2}\right)$ makes marketing effort, it is impossible for $S_{1}$ to benefit from a unilateral move to adopt a distribution strategy whether or not it makes marketing effort. That is, if its competitor sells products directly to consumers, $S_{1}$ may find the distribution strategy unprofitable, as shown in (a) of both Part (i) and Part (ii). This finding differs from Cachon and Harker (2002) because we consider the effect of marketing effort on demand. The competitor who adopts marketing effort and direct-sales strategy will be more competitive for gaining market shares. Second, when the competitor sells through a retailer, it is not always optimal for $S_{1}$ to sell 
directly if it is without marketing effort, as shown in (b) of Part (i) and Part (ii). If the competition coefficient is sufficiently high, the intense market competition stimulates demand, thus leading to an increase in order quantity. When faced with fierce price competition, each supplier benefits from the distribution channel strategy that mitigates retail competitiveness. Downstream retailers compete for consumers, whereas upstream suppliers are not directly involved in the competition for consumers. In contrast, when the market is mild, $S_{1}$ has no incentive to adopt the distribution channel strategy because the negative effect of double marginalization outweighs the positive effect on retail competition.

Our findings imply that when suppliers are faced with fierce price competition, a major role of distribution is to increase order quantity by enhancing the level of marketing effort, thus offsetting the impact of competition intensity from the retailers. This explains why, when faced with fierce retail competition, $S_{1}$ has the motivation to adopt a distribution strategy.

In the following, we will study how $S_{1}$ 's optimal channel structure strategy impacts the decisions and profits of the rival supplier who makes marketing effort. The results are presented in the following proposition:

Proposition 6 The strategic effects of $S_{1}$ 's motivation for channel structure selection are given as follows.

(i) When $S_{1}$ does not provide the marketing effort, we have
(a) $\left\{\begin{array}{l}p_{2}^{* C C} \leq p_{2}^{* D C} \\ q_{2}^{* C C}>q_{2}^{* D C}\end{array}\right.$ and $\Pi_{S 2}^{* C C} \leq \Pi_{S 2}^{* D C}$;
(b) $\left\{\begin{array}{l}p_{2}^{* D D} \geq p_{2}^{* C D} \\ q_{2}^{* D D} \geq q_{2}^{* C D}\end{array}\right.$ and $\Pi_{S 2}^{* D D} \geq \Pi_{S 2}^{* C D}$.

(ii) When $S_{1}$ provides the marketing effort, we have
(a) $\left\{\begin{array}{l}\tilde{p}_{2}^{* C C} \leq \tilde{p}_{2}^{* D C} \\ \tilde{q}_{2}^{* C C}>\tilde{q}_{2}^{* D C}\end{array}\right.$ and $\tilde{\Pi}_{S 2}^{* C C} \leq \tilde{\Pi}_{S 2}^{* D C}$;
(b) $\left\{\begin{array}{l}\tilde{p}_{2}^{* D D} \geq \tilde{p}_{2}^{* C D} \\ \tilde{q}_{2}^{* D D}<\tilde{q}_{2}^{* C D}\end{array}\right.$ and $\tilde{\Pi}_{S 2}^{* D D} \geq \tilde{\Pi}_{S 2}^{* C D}$.

Specifically, the above equalities are satisfied if and only if $\gamma=0$.

From Proposition 5, we believe that in both models, the direct-sales strategy is always optimal for $S_{1}$ when $S_{2}$ sells its products directly to consumers. However, according to Proposition 6 , we find that the direct-sales motivation of $S_{1}$ always hurts the profit of $S_{2}$ who sells its products directly to consumers, as shown in (a) of Parts (i) and (ii). In addition, if $S_{2}$ sells through a retailer, two outcomes are possible: (i) $S_{1}$ has the motivation to sell its products directly to customers under certain conditions, which will hurt $S_{2}$; (ii) $S_{1}$ also has the motivation to distribute its products, which will benefit $S_{2}$, as shown in (b) of Parts (i) and (ii). Figure 4 illustrates the supplier's channel structure strategies and their effects on the competitor's profitability.

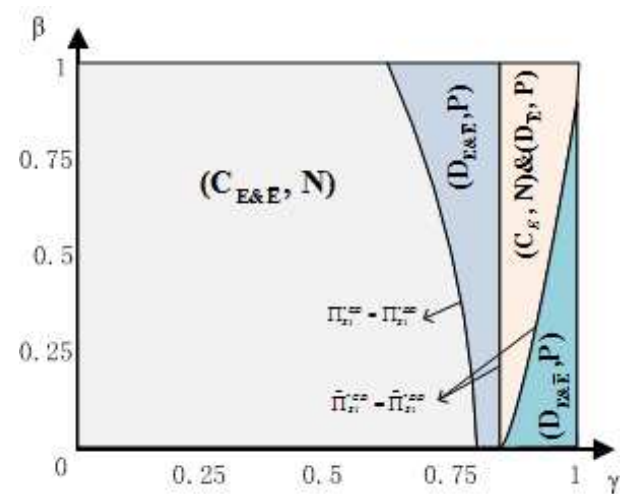

Fig. 4 Channel structure strategy and the strategic effects

$\left(C_{E \& \bar{E}}, N\right): S_{1}$ chooses to sell directly (C) and either makes marketing effort or not, which has negative effect on the competitor's profit;

$\left(D_{E \& \bar{E}}, P\right): S_{1}$ chooses to sell through a retailer (D) and either makes marketing effort or not, that has positive effect on the competitor's profit;

$\left(D_{\bar{E}}, P\right): S_{1}$ chooses to sell through a retailer (D) without marketing effort, which has positive effect on the competitor's profit;

$\left(C_{E}, N\right): S_{1}$ chooses to sell directly (C) with marketing effort, which has negative effect on the competitor's profit.

As shown in Figure 4, when retail competition is mild, $S_{1}$ prefers to sell its products directly to consumers, regardless of whether it makes marketing effort. This will compromise the competitor's profits. When retail competition is intense, however, $S_{1}$ 's channel structure strategy is determined by competition intensity as well as customers' sensitivity toward marketing effort. For example, when the market competition is very intense and the marketing effort sensitivity level is low, $S_{1}$ prefers to sell through a downstream retailer. 
From a perspective of strategic effects on the competitor, one can conclude that $S_{1}$ 's distribution channel (or direct-sales) strategy leads to a positive (or negative) effect on the competitor due to double marginalization. However, when considering the impacts of marketing effort motivation (see the subsection below), we find that the above conclusion changes.

\subsection{Motivations for Marketing Effort and Channel Structure Selection}

Here, we will simultaneously examine $S_{1}$ 's motivations toward marketing effort and channel structure selection, which we address in the following proposition.

Proposition 7 From $S_{1}$ 's perspective, we consider the following cases:

(i) When $S_{2}$ sells her products directly to consumers, we have

(a) If $(\gamma, \beta) \in\left\{0 \leq \gamma \leq 1,0 \leq \beta \leq \beta_{9}\right\}, \tilde{\Pi}_{S 1}^{* C C} \geq$ $\Pi_{S 1}^{* C C} \geq \tilde{\Pi}_{S 1}^{* D C}$; otherwise, $\tilde{\Pi}_{S 1}^{* C C} \geq \tilde{\Pi}_{S 1}^{* D C} \geq \Pi_{S 1}^{* C C}$;

(b) $\forall \gamma, \beta, \tilde{\Pi}_{S 1}^{* C C} \geq \Pi_{S 1}^{* D C}$.

(ii) When $S_{2}$ sells her products through a retailer, we have

if $(\gamma, \beta) \in\left\{0 \leq \gamma \leq \gamma_{6}, 0 \leq \beta \leq 1\right\} \cup\left\{\gamma_{6}<\right.$ $\left.\gamma \leq \gamma_{7}, 0 \leq \beta \leq \beta_{4}\right\} \cup\left\{\gamma_{8}<\gamma \leq 1, \beta_{5}<\right.$ $\beta \leq 1\}, \tilde{\Pi}_{S 1}^{* C D} \geq \max \left\{\Pi_{S 1}^{* C D}, \tilde{\Pi}_{S 1}^{* C D}, \Pi_{S 1}^{* \overline{D D}}\right\}$; otherwise, $\tilde{\Pi}_{S 1}^{* D D} \geq \max \left\{\Pi_{S 1}^{* D D}, \tilde{\Pi}_{S 1}^{* C D}, \Pi_{S 1}^{* C D}\right\}$, where $\beta_{9}$ is uniquely determined by $Z_{9}\left(\beta_{9} \mid \gamma\right)=0$.

The above equalities are satisfied if and only if $\beta=0$.

Proposition 7 states how $S_{1}$ 's marketing effort and channel structure options affect its order quantity, pricing as well as profits. Specifically, we have the following discussion.

(i) If $S_{1}$ has motivation for both marketing effort and channel structure selection, it is wise to first make a decision on marketing effort regardless of the competitor's channel structure selection.

(ii) When the competitor sells directly to consumers, we believe that $S_{1}$ has the motivation to make marketing effort and choose the direct-sales strategy based on the following findings: (a) $S_{1}$ must make marketing effort if it sells directly to consumers $\left(\Pi_{S 1}^{* C C}<\tilde{\Pi}_{S 1}^{* C C}\right)$, and it must choose to sell directly with the marketing effort $\left(\tilde{\Pi}_{S 1}^{* D C} \leq\right.$ $\tilde{\Pi}_{S 1}^{* C C}$ ); (b) if $S_{1}$ can choose any combination of marketing effort and channel structure strategies, we find that the combination of making marketing effort and selling directly is the optimal strategy $\left(\Pi_{S 1}^{* D C} \leq \tilde{\Pi}_{S 1}^{* C C}\right)$ due to high order quantity and low retail price. Therefore, we always have $\tilde{\Pi}_{S 1}^{* C C} \geq \max \left\{\Pi_{S 1}^{* C C}, \tilde{\Pi}_{S 1}^{* D C}, \Pi_{S 1}^{* D C}\right\}$.

(iii) When the competitor sells through a retailer, $S_{1}$ is motivated by marketing effort with the direct sales strategy under the conditions of $(\gamma, \beta) \in\left\{0 \leq \gamma \leq \gamma_{6}, 0 \leq \beta \leq 1\right\} \cup\left\{\gamma_{6}<\gamma \leq\right.$ $\left.\gamma_{7}, 0 \leq \beta \leq \beta_{4}\right\} \cup\left\{\gamma_{8}<\gamma \leq 1, \beta_{5}<\beta \leq 1\right\}$.

(iv) However, when the above conditions do not hold, we find that $S_{1}$ has the motivation to adopt the distribution channel strategy if the competitor chooses the same. The main reasons are as follows: (a) $S_{1}$ must make marketing effort if it adopts the distribution channel strategy $\left(\tilde{\Pi}_{S 1}^{* D D} \geq\right.$ $\Pi_{S 1}^{* D}$ ); (b) $S_{1}$ must choose the distribution channel strategy if it makes marketing effort $\left(\tilde{\Pi}_{S 1}^{* D D} \geq\right.$ $\tilde{\Pi}_{S 1}^{* C D}$ ); (c) if $S_{1}$ can choose any combination of marketing effort and channel structure strategies, its optimal strategy is making marketing effort and selling indirectly $\left(\tilde{\Pi}_{S 1}^{* D D} \geq \Pi_{S 1}^{* C D}\right)$. In this case, we find that the distribution channel strategy adopted by $S_{1}$ will result in a higher order quantity and retail price, see Proposition 3.

In summary, when $S_{1}$ has motivation for both marketing effort and channel structure selection, it is inclined to shift from not marketing effort to making marketing effort under any conditions, and from direct sales to indirect sales under certain conditions.

Proposition 8 The strategic effects of $S_{1}$ 's motivations towards marketing effort and channel structure selection are as follows.

(i) When $S_{2}$ sells her products directly to consumers, we have

$\left(S_{1}\right.$ : Switching from other options to "making marketing effort + direct sales"):

$\tilde{\Pi}_{S 2}^{* C C}\left\{\begin{array}{l}\geq \Pi_{S 2}^{* C C}, \forall \gamma, \beta \\ \leq \tilde{\Pi}_{S 2}^{* D C}, \forall \gamma, \beta \\ \leq \Pi_{S 2}^{* D C}, \forall \gamma, \beta\end{array}\right.$.

(ii) When $S_{2}$ sells her products through a retailer, we have

$\left(S_{1}\right.$ : Switching from other options to "making marketing effort + direct sales"):

$\tilde{\Pi}_{S 2}^{* C D}\left\{\begin{array}{l}\geq \Pi_{S 2}^{* C D}, \forall \gamma, \beta \\ \leq \tilde{\Pi}_{S 2}^{* D} D, \forall \gamma, \beta ; \\ \leq \Pi_{S 2}^{* D},, \forall \gamma, \beta\end{array} ;\right.$

$\left(S_{1}\right.$ : Switching from other options to "making marketing effort + indirect sales"): 


$$
\tilde{\Pi}_{S 2}^{* D D}\left\{\begin{array}{l}
\geq \Pi_{S 2}^{* C D}, \forall \gamma, \beta \\
\geq \tilde{\Pi}_{S 2}^{* C D}, \forall \gamma, \beta \\
\left.\geq \Pi_{S 2}^{* D D}, \quad \cup \gamma, \beta\right) \in\left\{0 \leq \gamma \leq \gamma_{12}, 0 \leq \beta \leq 1\right\} \\
\quad \cup\left\{\gamma_{12}<\gamma \leq 1,0 \leq \beta \leq \beta_{8}\right\} .
\end{array}\right.
$$

From Proposition 8, we find that $S_{1}$ 's decision on making marketing effort and choosing direct sales has both positive and negative effects on the competitor's profits when the competitor sells directly to consumers. For example, $S_{1}$ 's strategy to shift from distribution to direct sales $\left(\tilde{\Pi}_{S 2}^{* C C} \leq \tilde{\Pi}_{S 2}^{* D C}\right)$ hurts the competitor's profit. But $S_{1}$ 's strategy to shift from making no marketing effort to making them $\left(\tilde{\Pi}_{S 2}^{* C C} \geq \Pi_{S 2}^{* C C}\right)$ benefits the competitor. The first part implies that $S_{1}$ 's centralized decision weakens the competitor's marketing power. The second part indicates that the marketing effort will absorb the total market demand, which will benefit both suppliers. In addition, when $S_{1}$ switches from a "distribution channel without marketing effort" option to a "direct sales with marketing effort" option, the competitor's profits will be negatively affected $\left(\tilde{\Pi}_{S 2}^{* C C} \leq \Pi_{S 2}^{* D C}\right)$. When the competitor sells through a retailer, we find similar insights into the effects of the supplier's "direct sales with marketing effort" option on the competitor's profits. However, the competitor may benefit from $S_{1}$ 's "distribution channel with marketing effort" option under certain conditions. Figure 5 illustrates $S_{1}$ 's marketing effort motivation and channel structure strategy and their effects on the competitor's profitability.

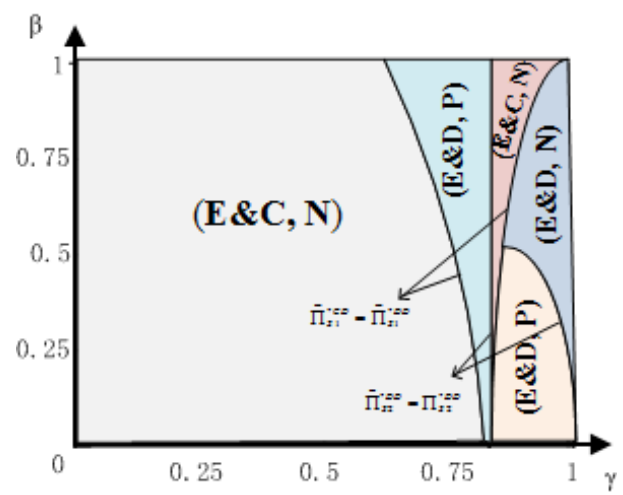

Fig. 5 Marketing effort motivation, channel structure strategy and the strategic effects
$(E \& C, N): S_{1}$ makes marketing effort and sells to market directly (C), which has negative effects on the competitor's profit;

$(E \& D, P): S_{1}$ makes marketing effort and sells through a retailer (D), which has positive effects on the competitor's profit;

$(E \& D, N): S_{1}$ makes marketing effort and sells through a retailer (D), which has negative effects on the competitor's profit;

If $S_{1}$ has options regarding a channel structure and marketing effort, we find that $S_{1}$ is motivated to make marketing effort as reflected in Figure 5. From a perspective of channel structure selection, $S_{1}$ may prefer to sell its products directly to consumers in the regions $(E \& C, N)$, identified by $\tilde{\Pi}_{S 1}^{* C D}>\tilde{\Pi}_{S 1}^{* D D} . S_{1}$ may adopt the distribution strategy in the other regions, i.e., $(E \& D, P)$ and $(E \& D, N)$. Some of our insights are different from previous research (Atkins and Liang 2010, McGuire and Staelin 1983), which argues that when faced with fierce retail competition, a supplier may prefer to add an intermediary (retailer) to avoid direct competition with another supplier. Considering the effects of marketing effort on profitability, we find that under fierce retail competition: (i) when consumers' marketing effort sensitivity level is low, the supplier adopts a distribution strategy because it suppresses retail competition $(E \& D)$; (ii) when consumers' marketing effort sensitivity level is sufficiently high, the supplier prefers to sell products to consumers directly $(E \& C)$. While this may sound counterintuitive, when the market is highly competitive, the supplier has the power to compete with its competitor by raising its marketing effort. Finally, under certain market conditions (e.g., high retail competition pressure and moderate customer's marketing effort sensitivity level), $S_{1}$ 's distribution decision can attract more customers due to price advantage, thus, negatively affecting the competitor $\left(S_{2}\right)$, as shown in the region of $(E \& D, N)$. In reality, under high competition pressure, the supplier who sells through a retailer and increases its marketing effort attracts more customers from the competitor, thus leading to a negative effect on the competitor's profitability. 


\section{Conclusions}

This paper investigates and compares the economic impacts of different channel structures using a simple model of two competing suppliers selling their own products by direct-sales or distribution channel. We explore the channel structure selection and marketing effort motivations of the supplier and their strategic effects on the competitor's profitability. First, in the case that both suppliers make marketing effort, we develop a game theoretic model to explore how the channel structure strategies can reach equilibria. Unlike most previous studies on channel analysis that focus on one given channel structure, our game theoretic approach aims to solve the equilibrium channel structure under different market conditions (e.g., marketing effort level and retail competition intensity). Whether or not the supplier makes marketing effort, we find that the direct-sales strategy (i.e., CC channel structure) is a dominant equilibrium strategy for both the supplier and the competitor. In addition, under fierce price competition, we find that supplier and competitor may benefit from a distribution strategy that mitigates retail competitiveness, i.e., the DD structure which is another equilibrium channel structure. Different from Cachon and Harker (2002), we find that when the competitor makes marketing effort, it is impossible for the supplier to benefit from a unilateral move to a distribution channel.

Our work also focuses on the supplier's motivation for making marketing effort, which depends on not only channel structure but also market conditions. For example, under low competition pressure and high consumers' sensitivity towards marketing effort level, the supplier is not motivated to make marketing effort when she distributes products and her competitor sells directly. When the competitor adopts a distribution channel strategy, however, the supplier always has the motivation to make marketing effort. Further, we explore how the supplier adjusts her decisions according to the various market conditions when she adopts both marketing effort and channel structure options. We find that in this case the supplier always makes marketing effort. Under intense retail competition and low customer's marketing effort sensitivity level, the supplier will choose the distribution channel to suppress price competition. However, if the customer's sensitivity level is sufficiently high, the supplier prefers to sell products to the market directly. These findings distinguish our study from traditional research on supply chain competition.

Finally, we investigate how the supplier's strategies of marketing effort and channel structure selection affect the competitor's profitability. We find that the supplier's marketing effort motivation does not always have a positive impact on competitors. Additionally, the supplier's channel structure selection has different effects on the competitor's profit. In most cases, the supplier's distribution channel (direct-sales) strategy can lead to a positive (negative) effect on the competitor's profitability. However, we believe that the supplier adopting the distribution channel strategy can lead to a negative effect on the competitor's profit when considering the motivations to make marketing effort under certain market conditions.

Our research lays the foundation for examining the supplier's marketing effort and channel structure decisions, and could continue in several directions. For example, our paper mainly discusses marketing effort and channel structure decisions in competitive supply chains and describes two kinds of models and four kinds of channel structures. Further research can study more sophisticated supply chain management scenarios that, for example, take contractual issues into account. In addition, it will be interesting to see if the results of our study are likely to change, when the retailers in the competitive supply chains focus on their own profits. For mathematical tractability, our study does not consider the marketing effort competition between suppliers. Future research may resort to numerical analysis to examine both retail and marketing effort competition for meaningful managerial insight.

Acknowledgments. This work was supported by National Natural Science Foundation of China [Grant number 71971113].

Data availability. Data sharing not applicable to this article as no datasets were generated or analysed during the current study. 


\section{Appendix A Symbols and functions}

A.1. The following symbols are used to simplify the results, where $i=1,2$ and $i \neq j$.

$$
\begin{aligned}
& A=(2+\gamma) a+(2+\gamma)(\gamma-1) c ; \\
& B=(2+\gamma) a+\left(2-\gamma^{2}\right) c ; \\
& S=2 \gamma^{2}+\gamma-4 ; \\
& T=2 \gamma^{2}-4-\gamma ; \\
& \tau\left(t_{i}, t_{j}\right)=(2+\gamma) a+2 \beta \sqrt{t_{i}}+\beta \gamma \sqrt{t_{j}} ; \\
& \phi=4\left(4-\gamma^{2}\right)+\left(\gamma^{2}-4\right) \beta^{2} ; \\
& \varphi=2\left(4-2 \gamma^{2}+\gamma\right)+(\gamma-2) \beta^{2} ; \\
& M=4\left(2-\gamma^{2}\right)\left(4-\gamma^{2}\right)-\left(8-3 \gamma^{2}\right) \beta^{2} ; \\
& N=2\left(4-\gamma^{2}\right)+(\gamma-2) \beta^{2} .
\end{aligned}
$$

A.2. The solutions $\beta_{i}$ and $\gamma_{j}$ presented in all propositions and corollaries are determined by $Z_{i}\left(\beta_{i} \mid \gamma_{j}\right)=0$, where the functions $Z_{i}\left(\beta_{i} \mid \gamma_{j}\right)$ are given as

$Z_{1}\left(\beta_{1} \mid \gamma\right)=4\left(4-\gamma^{2}\right)^{1 / 2}\left(2-\gamma^{2}\right)^{3 / 2}-2\left(4 \gamma^{4}-\right.$ $\left.17 \gamma^{2}+16\right)+\left[8-3 \gamma^{2}-2\left(4-\gamma^{2}\right)^{1 / 2}\left(2-\gamma^{2}\right)^{1 / 2}\right] \beta^{2}$; $Z_{2}\left(\beta_{2} \mid \gamma\right)=\left[4\left(2-\gamma^{2}\right)-\left(4-\gamma^{2}\right) \beta^{2}\right] M^{2}-$ $\phi\left[2 S T-\left(8-3 \gamma^{2}\right) \beta^{2}\right]^{2}$;

$Z_{3}\left(\beta_{3} \mid \gamma\right)=2\left(\gamma^{2}-4\right) S T+8\left(8-3 \gamma^{2}\right)\left(2-\gamma^{2}\right)-$ $\left(4-\gamma^{2}\right)\left(8-3 \gamma^{2}\right) \beta^{2}$;

$Z_{4,5}\left(\beta_{4}, \beta_{5} \mid \gamma\right)=\left[4\left(2-\gamma^{2}\right)-(4-\right.$ $\left.\left.\gamma^{2}\right) \beta^{2}\right]\left(2 M-\phi \beta^{2}\right)^{2}-\varphi^{2}\left(4-\beta^{2}\right)(4-$ $\left.\gamma^{2}\right)\left[2\left(\gamma+2 \gamma^{2}-4\right)+(\gamma+2) \beta^{2}\right]^{2}$;

$Z_{6}\left(\beta_{6} \mid \gamma\right)=\left[2 \gamma(2 \gamma-3)+(2-\gamma) \beta^{2}\right]\left[2\left(2 \gamma^{2}-4+\right.\right.$ $\left.\gamma)+(2+\gamma) \beta^{2}\right]$

$Z_{7}\left(\beta_{7} \mid \gamma\right)=-\left(2-\gamma^{2}\right)\left(4-\gamma^{2}\right) \beta^{6}+\left(64-56 \gamma^{2}+\right.$ $\left.11 \gamma^{4}\right) \beta^{4}+8\left(2-\gamma^{2}\right)\left(\gamma^{4}-8\right) \beta^{2}+16 \gamma^{2}\left(2-\gamma^{2}\right)^{2}(4-$ $\left.\gamma^{2}\right)$;

$Z_{8}\left(\beta_{8} \mid \gamma\right)=2\left(2 \gamma^{2}-4+\gamma\right)\left(2 \gamma^{2}-4-\gamma\right)\left(3-\gamma^{2}\right)+$ $(\gamma-3)\left(-\gamma^{3}-3 \gamma^{2}+3 \gamma+8\right) \beta^{2}$;

$Z_{9}\left(\beta_{9} \mid \gamma\right)=2\left(4-\gamma^{2}\right)\left(4-\gamma^{2}-\beta^{2}\right)^{2}\left[8-4 \gamma^{2}-\right.$ $\left.\left(4-\gamma^{2}\right) \beta^{2}\right]-\left[2 M-\phi \beta^{2}\right]^{2}$.

\section{Appendix B Proofs}

Proof of Theorem 1: For the four channel structures under Model I, we give the proof as follows.

(i) Channel structure CC

The demands of $S_{1}$ and $S_{2}$ are $q_{1}=a-p_{1}+\gamma p_{2}$ and $q_{2}=a-p_{2}+\gamma p_{1}+\beta \sqrt{e_{2}}$ respectively. The profit functions of $S_{1}$ and $S_{2}$ can be presented by $\Pi_{S 1}^{C C}=\left(p_{1}-c\right) q_{1}$ and $\Pi_{S 2}^{C C}=\left(p_{2}-c\right) q_{2}-e_{2}$ respectively.

The optimization problem of chains $\mathrm{i}$ is $\max _{p_{i}} \Pi_{S i}^{C C}\left(p_{i}, p_{j}\right)$. Because $\partial \Pi_{S 1}^{C C} / \partial p_{1}=a-2 p_{1}+$ $\gamma p_{2}+c$ and $\partial^{2} \prod_{S 1}^{C C} / \partial p_{1}{ }^{2}=-2<0, \Pi_{S 1}^{C C}$ is concave over $p_{1}$. Similarly, we can show that $\Pi_{S 1}^{C C}$ is concave over $p_{2}$. From the first-order conditions, we have

$$
\begin{aligned}
& \partial \Pi_{S 1}^{C C} / \partial p_{1}=a-2 p_{1}+\gamma p_{2}+c=0 \text { and } \\
& \partial \Pi_{S 2}^{C C} / \partial p_{2}=a-2 p_{2}+\gamma p_{1}+\beta \sqrt{e_{2}}+c=0 .
\end{aligned}
$$

Because $\partial^{2} \Pi_{S 2}^{C C} / \partial e_{2}{ }^{2}<0, \Pi_{S 2}^{C C}$ is concave over $e_{2}$ and we have

$$
\partial \Pi_{S 2}^{C C} / \partial e_{2}=\left(p_{2}-c\right) \beta /\left(2 \sqrt{e_{2}}\right)-1=0 .
$$

From Eqs. (B1) and (B2), the equilibrium solutions of decision and profit are given by Table 1 .

(ii) Channel structure CD

The demand of $S_{1}$ is $q_{1}=a-p_{1}+\gamma p_{2}$. The profit function of $S_{1}$ is $\Pi_{S 1}^{C D}=\left(p_{1}-c\right) q_{1}$. The optimization problem is $\max _{p_{1}} \Pi_{S 1}^{C D}\left(p_{1}, p_{2}\right)$. Similarly to channel structure $\mathrm{CC}$, we can prove that the function $\Pi_{S 1}^{C D}$ is concave over the price $p_{1}$. We have

$$
\partial \Pi_{S 1}^{C D} / \partial p_{1}=a-2 p_{1}+\gamma p_{2}+c=0 .
$$

The demand of $S_{2}$ is $q_{2}=a-p_{2}+\gamma p_{1}+\beta \sqrt{e_{2}}$. The profit function of $R_{2}$ is $\Pi_{R 2}^{C D}=\left(p_{2}-w_{2}\right) q_{2}$. Given the unit wholesale price $w_{2}$ offered by its upstream $S_{2}, R_{2}$ 's optimal problem is written as $\max _{p_{2}} \Pi_{R 2}^{C D}\left(p_{2} \mid w_{2}, p_{1}\right)$. Obviously, $\partial \Pi_{R 2}^{C D} / \partial p_{2}=$ $a-2 p_{2}+\gamma p_{1}+\beta \sqrt{e_{2}}+w_{2}$ and $\partial^{2} \Pi_{R 2}^{C D} / \partial p_{2}{ }^{2}=$ $-2<0$, therefore, we have $\Pi_{R 2}^{C D}$ is concave over $p_{2}$. We have

$$
\partial \Pi_{R 2}^{C D} / \partial p_{2}=a-2 p_{2}+\gamma p_{1}+\beta \sqrt{e_{2}}+w_{2}=0 .
$$

From Eqs. (B3) and (B4), we can derive

$$
\begin{aligned}
& p_{1}^{* C D}=\frac{(2+\gamma) a+2 c+\beta \gamma \sqrt{e_{2}^{C D}}+\gamma w_{2}^{C D}}{4-\gamma^{2}}, \\
& p_{2}^{* C D}=\frac{(2+\gamma) a+2 c+2 \beta \sqrt{e_{2}^{C D}}+2 w_{2}^{C D}}{4-\gamma^{2}} .
\end{aligned}
$$

The profit function of $S_{2}$ is $\Pi_{S 2}^{C D}=\left(w_{2}-c\right) q_{2}-e_{2}$. According to Eqs. (B5) and (B6), the optimization problem of $S_{2}$ is $\max _{w_{2}} \Pi_{S 2}^{C D}\left(w_{2}, p_{2}, p_{1}\right)$.

From $\partial \Pi_{S 2}^{C D} / \partial w_{2}=\left[(2+\gamma) a+2 \beta \sqrt{e_{2}}+\left(2 \gamma^{2}-\right.\right.$ 4) $\left.w_{2}+\left(\gamma-\gamma^{2}+2\right) c\right] /\left(4-\gamma^{2}\right)$ and $\partial^{2} \Pi_{S 2}^{C D} / \partial w_{2}{ }^{2}=$ 
$2 \gamma^{2}-4<0$, the function $\Pi_{S 2}^{C D}$ is concave over $w_{2}$. Then, from the first-order condition, we have

$$
\begin{aligned}
\partial \Pi_{S 2}^{C D} / \partial w_{2} & =\frac{(2+\gamma) a+2 \beta \sqrt{e_{2}}+\left(2 \gamma^{2}-4\right) w_{2}}{+\left(\gamma-\gamma^{2}+2\right) c} \\
& =0
\end{aligned}
$$

It is difficult to obtain the optimal solution when substituting Eqs. (B5) and (B6) into $\Pi_{S 2}^{C D}$, and we then consider an approximate optimal solution for any wholesale/retail price. Since $\partial^{2} \prod_{S 2}^{C D} / \partial e_{2}{ }^{2}<$ 0 , the optimal marketing effort level of $S_{2}$ should satisfy the first-order condition for the given prices, which is written as follows.

$$
\left(w_{2}-c\right) \beta /\left(2 \sqrt{e_{2}}\right)-1=0 .
$$

From Eqs. (B7) and (B8), we can derive the equilibrium solutions as Table 1 showing.

(iii) Channel structure DC

The demand of $S_{1}$ is $q_{1}=a-p_{1}+\gamma p_{2}$. The profit function of $R_{1}$ is $\Pi_{R 1}^{D C}=\left(p_{1}-w_{1}\right) q_{1}$. Given the unit wholesale price determined by the upstream $S_{1}$, the optimal problem of $R_{1}$ is $\max _{p_{1}} \Pi_{R 1}^{D C}\left(p_{1} \mid w_{1}, p_{2}\right)$. Since $\partial \prod_{R 1}^{D C} / \partial p_{1}=a-$ $2 p_{1}+\gamma p_{2}+w_{1}$ and $\partial^{2} \Pi_{R 1}^{D C} / \partial p_{1}{ }^{2}=-2<0$, we have that the function $\Pi_{R 1}^{D C}$ is concave over $p_{1}$. From the first-order condition of $\partial \Pi_{R 1}^{D C} / \partial p_{1}$, we have

$$
a-2 p_{1}+\gamma p_{2}+w_{1}=0 .
$$

The demand of $S_{2}$ is $q_{2}=a-p_{2}+\gamma p_{1}+\beta \sqrt{e_{2}}$. The profit of $S_{2}$ is written as $\max _{p_{2}} \Pi_{S 2}^{D C}=\left(p_{2}-c\right) q_{2}$. Similarly, we can show that $\Pi_{S 2}^{D C}$ is concave over $p_{2}$. We have

$$
\partial \Pi_{S 2}^{D C} / \partial p_{2}=a-2 p_{2}+\gamma p_{1}+\beta \sqrt{e_{2}}+c=0 .
$$

From Eqs. (B9) and (B10), we can derive the following results.

$$
\begin{aligned}
& p_{1}^{* D C}=\frac{(2+\gamma) a+\gamma c+\beta \gamma \sqrt{e_{2}^{D C}}+2 w_{1}^{D C}}{4-\gamma^{2}}, \\
& p_{2}^{* D C}=\frac{(2+\gamma) a+2 c+2 \beta \sqrt{e_{2}^{D C}}+\gamma w_{2}^{D C}}{4-\gamma^{2}} .
\end{aligned}
$$

According to Eqs. (B11) and (B12), the optimal problem of $S_{1}$ is written as $\max _{w_{1}} \Pi_{S 1}^{D C}\left(w_{1}, p_{1}, p_{2}\right)$. Since $\frac{\partial \Pi_{S 1}^{D C}}{\partial w_{1}}=$

$\frac{(2+\gamma) a+\gamma \beta \sqrt{e_{2}}+2\left(\gamma^{2}-2\right) w_{1}+\left(\gamma-\gamma^{2}+2\right) c}{4-\gamma^{2}}$ and $\frac{\partial^{2} \Pi_{S 1}^{D C}}{\partial w_{1}^{2}}=2\left(\gamma^{2}-2\right)<0$, the function $\Pi_{S 1}^{D C}$ is concave over $w_{1}$. From the first-order condition, we have

$$
w_{1}^{* D C}=\frac{(2+\gamma) a+\left(\gamma-\gamma^{2}+2\right) c+\gamma \beta \sqrt{e_{2}^{D C}}}{4-2 \gamma^{2}} .
$$

Similarly, the optimal problem of $S_{2}$ is $\max _{e_{2}} \Pi_{S 2}^{D C}\left(e_{2}, p_{1}, p_{2}\right)$. Since $\partial^{2} \Pi_{S 2}^{D C} / \partial e_{2}{ }^{2}<0$, the optimal marketing effort level of $S_{2}$ should satisfy the first-order condition $\partial \Pi_{S 2}^{D C} / \partial e_{2}=0$ for the given prices and we have

$$
e_{2}^{* D C}=\left(p_{2}^{D C}-c\right)^{2} \beta^{2} / 4 .
$$

According to Eqs. (B12), (B13) and (B14), we can derive the equilibrium solutions as Table 1 showing.

(iv) Channel structure DD

The demand of $S_{1}$ is $q_{1}=a-p_{1}+\gamma p_{2}$ and that of $S_{2}$ is $q_{2}=a-p_{2}+\gamma p_{1}+\beta \sqrt{e_{2}}$. The profit functions of $S_{1}$ and $R_{1}$ are $\Pi_{S 1}^{D D}=\left(p_{1}-w_{1}\right) q_{1}$ and $\Pi_{R 1}^{D D}=\left(w_{1}-c\right) q_{1}$ respectively. Similarly, it is easy to show that $\Pi_{S 1}^{D D}$ is concave over $p_{1}$ and $\Pi_{R 1}^{D D}$ is concave over $w_{1}$. Given $w_{1}$ offered by the upstream $S_{1}, R_{1}$ 's optimal problem is $\max _{p_{1}} \Pi_{R 1}^{D D}\left(p_{1}, w_{1}, p_{2}\right)$. We have

$$
\partial \Pi_{R 1}^{D D} / \partial p_{1}=a-2 p_{1}+\gamma p_{2}+w_{1}=0 .
$$

For supply chain 2, the profit functions of $S_{2}$ and $R_{2}$ are $\Pi_{S 2}^{D D}=\left(p_{2}-w_{2}\right) q_{2}-e_{2}$ and $\Pi_{R 2}^{D D}=\left(w_{2}-\right.$ c) $q_{2}$ respectively. Similarly, it is easy to show $\Pi_{S 2}^{D D}$ and $\Pi_{R 2}^{D D}$ are concave over $p_{2}$ and $w_{2}$ respectively. Given the unit wholesale price $\left(w_{2}\right)$ determined by the upstream $S_{2}$, the optimal problem of $R_{2}$ is $\max _{p_{2}} \Pi_{R 2}^{D D}\left(p_{2}, w_{2}, p_{1}\right)$. We have

$$
\partial \Pi_{R 2}^{D D} / \partial p_{2}=a-2 p_{2}+\gamma p_{1}+\beta \sqrt{e_{2}}+w_{2}=0 .
$$


From Eqs. (B15) and (B16) we can obtain the best responses as follows.

$$
\begin{aligned}
& p_{1}^{* D D}=\frac{(2+\gamma) a+\gamma \beta \sqrt{e_{2}^{D D}}+2 w_{1}^{D D}+\gamma w_{2}^{D D}}{4-\gamma^{2}}, \\
& p_{2}^{* D D}=\frac{(2+\gamma) a+2 \beta \sqrt{e_{2}^{D D}}+2 w_{2}^{D D}+\gamma w_{1}^{D D}}{4-\gamma^{2}} .
\end{aligned}
$$

Based on Eqs. (B17) and (B18), the profit of $S_{i}$ is written as $\max _{w_{i}} \Pi_{S i}^{D D}\left(w_{i} \mid p_{i}, p_{j}\right)$, where $i, j=1,2$ and $i \neq j$. We have $\partial \Pi_{S 1}^{D D} / \partial w_{1}=$ $\left[a-p_{1}+\gamma p_{2}+\left(\gamma^{2}-2\right)\left(w_{1}-c\right)\right] /\left(4-\gamma^{2}\right)=0$ and $\partial \Pi_{S 2}^{D D} / \partial w_{2}=\left[a-p_{2}+\gamma p_{1}+\right.$ $\left.\beta \sqrt{e_{2}}+\left(\gamma^{2}-2\right)\left(w_{2}-c\right)\right] /\left(4-\gamma^{2}\right)=0$. Therefore, we obtain

$$
\begin{aligned}
& w_{1}^{* D D}=\left[2 \gamma\left(3-\gamma^{2}\right) \beta \sqrt{e_{2}^{D D}}-T B\right] /(S T), \\
& w_{2}^{* D D}=\left[\left(8-3 \gamma^{2}\right) \beta \sqrt{e_{2}^{D D}}-T B\right] /(S T) .
\end{aligned}
$$

Similar to (ii), we decide the optimal marketing effort level of $S_{2}$ according to the first-order condition (i.e., $\partial \Pi_{S 2}^{D D} / \partial e_{2}=\left(w_{2}-c\right) \beta /\left(2 \sqrt{e_{2}}\right)-1=0$ ) and have

$$
e_{2}^{* D D}=\left(w_{2}^{D D}-c\right)^{2} \beta^{2} / 4 .
$$

From Eqs. (B19-21), we can derive the equilibrium solutions as Table 1.

\section{Proof of Corollary 1:}

(i) From Theorem 1, we have

$$
p_{1}^{* C C}-p_{2}^{* C C}=\left\{\left(\beta^{2}-4-2 \gamma\right) a+\left(\beta^{2}-4-2 \gamma+\right.\right.
$$
$\left.\left.\gamma \beta^{2}\right) c+2\left[(2+\gamma) a+\left(2+\gamma-\beta^{2}\right) c\right]\right\} /\left[2\left(\gamma^{2}+\beta^{2}-4\right)\right]$.

Here, we have $2\left(\gamma^{2}+\beta^{2}-4\right)<0$, and $\left(\beta^{2}-\right.$ $4-2 \gamma) a+\left(\beta^{2}-4-2 \gamma+\gamma \beta^{2}\right) c+2[(2+\gamma) a+$ $\left.\left(2+\gamma-\beta^{2}\right) c\right]=\beta^{2}[a+(\gamma-1) c]$. Meanwhile, since $\beta, \gamma \in[0,1]$, we obtain $\beta^{2}[a+(\gamma-1) c] \geq 0$ and $\beta^{2}[a+(\gamma-1) c]=0$ if and only if $\beta=0$. Therefore we have $p_{1}^{* C C} \leq p_{2}^{* C C}$.

Similarly, from Theorem 1 , we have $q_{1}^{* C C}-$ $q_{2}^{* C C}=\beta^{2}[a+(\gamma-1) c] /\left[2\left(\gamma^{2}+\beta^{2}-4\right)\right]$. It is easy to find $q_{1}^{* C C}-q_{2}^{* C C} \leq 0$. Obviously, $q_{1}^{* C C}-q_{2}^{* C C}=0$ if and only if $\beta=0$.

Meanwhile, from Theorem 1, we have

$\Pi_{S 1}^{* C C}-\Pi_{S 2}^{* C C}$

$=A^{2}\left(\beta^{2}-4-2 \gamma\right)^{2} /\left[4(2+\gamma)^{2}\left(\gamma^{2}+\beta^{2}-4\right)^{2}\right]$

$-A^{2}\left(4-\beta^{2}\right) /\left[4\left(\gamma^{2}+\beta^{2}-4\right)^{2}\right]$

$=\beta^{2}\left(\gamma^{2}+\beta^{2}-4\right) A^{2} /\left[4(2+\gamma)^{2}\left(\gamma^{2}+\beta^{2}-4\right)^{2}\right]$.

Because $\beta, \gamma \in[0,1]$, we have $\Pi_{S 2}^{* C C} \geq \Pi_{S 1}^{* C C}$. Obviously, $\Pi_{S 2}^{* C C}=\Pi_{S 1}^{* C C}$ if and only if $\beta=0$.
From Theorem 1, we have

$$
\begin{aligned}
& p_{1}^{* D D}-p_{2}^{* D D} \\
= & \frac{(2+\gamma) a+\gamma \beta \sqrt{e_{2}^{* D D}}+2 w_{1}^{* D D}+\gamma w_{2}^{* D D}}{4-\gamma^{2}} \\
- & \frac{(2+\gamma) a+2 \beta \sqrt{e_{2}^{* D D}}+2 w_{2}^{* D D}+\gamma w_{1}^{* D D}}{4-\gamma^{2}} \\
= & \frac{\left(4 \gamma^{4}+2 \gamma^{3}-6 \gamma-20 \gamma^{2}+24\right) \beta \sqrt{e_{2}^{* D D}}}{\gamma^{2}-\left(2 \gamma^{2}-4\right)^{2}} .
\end{aligned}
$$

Because $\beta, \gamma \in[0,1]$, we have $p_{1}^{* D D} \leq p_{2}^{* D D}$. Obviously, $p_{1}^{* D D}=p_{2}^{* D D}$ if and only if $\beta=0$.

Similarly, from Theorem 1, we have

$$
\begin{aligned}
& =\frac{\left(2-\gamma^{2}\right)\left[T A+2\left(\gamma^{2}-3\right) \gamma \beta \sqrt{\left.e_{2}^{* D D}\right]}\right.}{\left(\gamma^{2}-4\right) S T} \\
& -\frac{\left(2-\gamma^{2}\right)\left[T A+\left(3 \gamma^{2}-8\right) \beta \sqrt{\left.e_{2}^{* D D}\right]}\right.}{\left(\gamma^{2}-4\right) S T}
\end{aligned}
$$$$
=\frac{\left(2-\gamma^{2}\right) \beta \sqrt{e_{2}^{* D D}}}{\left(\gamma^{2}-4\right) S T} \text {. }
$$

Because $\beta, \gamma \in[0,1]$ and $S T>0$, we have $q_{1}^{* D D} \leq q_{2}^{* D D}$. Obviously, $q_{1}^{* D D}=q_{2}^{* D D}$ if and only if $\beta=0$.

Moreover, from Theorem 1, we have $w_{1}^{* D D}-w_{2}^{* D D}$

$=\left[2 \gamma\left(3-\gamma^{2}\right) \beta \sqrt{e_{2}^{* D D}}-T B\right] /(S T)$

$-\left[\left(8-3 \gamma^{2}\right) \beta \sqrt{e_{2}^{* D D}}-T B\right] /(S T)$

$=\left(-2 \gamma^{3}+3 \gamma^{2}+6 \gamma-8\right) \beta \sqrt{e_{2}^{* D D}} /(S T)$.

Because $\beta, \gamma \in[0,1]$ and $S T>0$, we have $w_{1}^{* D D} \leq w_{2}^{* D D}$. Obviously, $w_{1}^{* D D}=w_{2}^{* D D}$ if and only if $\beta=0$.

Meanwhile, we can prove $\Pi_{S 1}^{* D D} \leq \Pi_{S 2}^{* D D}$ by the similar method.

(ii) From Theorem 1, we have

$$
p_{1}^{* C D}-p_{2}^{* C D}
$$

$=\left[(2+\gamma) a+2 c+\gamma \beta \sqrt{e_{2}^{* C D}}+\gamma w_{2}^{* C D}\right] /\left(4-\gamma^{2}\right)$

$-\left[(2+\gamma) a+\gamma c+2 \beta \sqrt{e_{2}^{* C D}}+2 w_{2}^{* C D}\right] /\left(4-\gamma^{2}\right)$

$=\left[-A+\left(2 \gamma^{2}-6\right) \beta \sqrt{e_{2}^{* C D}}\right] /\left[2\left(2-\gamma^{2}\right)(2+\gamma)\right]$.

Because $\beta, \gamma \in[0,1]$, we have $p_{1}^{* C D} \leq p_{2}^{* C D}$. Obviously, $p_{1}^{* C D}=p_{2}^{* C D}$ if and only if $\beta=0$.

Meanwhile, from Theorem 1, we have

$q_{1}^{* C D}-q_{2}^{* C D}$

$$
\begin{aligned}
& =\frac{\varphi^{2} A^{2}}{\left(4-\gamma^{2}\right)^{2}\left[4\left(2-\gamma^{2}\right)-2 \beta^{2}\right]^{2}} \\
& -\frac{\left[4\left(2-\gamma^{2}\right)-\left(4-\gamma^{2}\right) \beta^{2}\right] A^{2}}{\left(4-\gamma^{2}\right)^{2}\left[4\left(2-\gamma^{2}\right)-2 \beta^{2}\right]^{2}} \\
& =\frac{(2-\gamma)\left[2(\gamma+1)-\beta^{2}\right] A}{\left(4-\gamma^{2}\right)^{2}\left[4\left(2-\gamma^{2}\right)-2 \beta^{2}\right]^{2}} .
\end{aligned}
$$


Because $\beta, \gamma \in[0,1]$ and $A>0$, then we have $q_{1}^{* C D} \geq q_{2}^{* C D}$.

From Theorem 1, we have

$p_{1}^{* D C}-p_{2}^{* D C}$

$=\left[(2+\gamma) a+\gamma c+\gamma \beta \sqrt{e_{2}^{* D C}}+2 w_{1}^{* D C}\right] /\left(4-\gamma^{2}\right)$

$-\left[(2+\gamma) a+2 c+2 \beta \sqrt{e_{2}^{* D C}}+\gamma w_{1}^{* D C}\right] /\left(4-\gamma^{2}\right)$

$=\left(w_{1}^{* D C}-c-\beta \sqrt{e_{2}^{* D C}}\right) /(2+\gamma)$.

It is easy to find that $p_{1}^{* D C} \geq p_{2}^{* D C}$ is satisfied if and only if $w_{1}^{* D C}-c \geq \beta \sqrt{e_{2}^{* D C}}$ is satisfied. Then we have $w_{1}^{* D C}-c-\beta \sqrt{e_{2}^{* D C}}=$ $\left[A+\left(\gamma-4+2 \gamma^{2}\right) \beta \sqrt{e_{2}^{* D C}}\right] /\left(4-2 \gamma^{2}\right)$.

Since $4-2 \gamma^{2}>0$, we find $w_{1}^{* D C}-c \geq \beta \sqrt{e_{2}^{* D C}}$ is equivalent to $A+\left(\gamma-4+2 \gamma^{2}\right) \beta \sqrt{e_{2}^{* D C}} \geq 0$.

Then, we have $A+\left(\gamma-4+2 \gamma^{2}\right) \beta \sqrt{e_{2}^{* D C}}=$ $\left[4\left(2-\gamma^{2}\right)\left(4-\gamma^{2}\right)+\left(20 \gamma^{2}-24-4 \gamma^{4}\right) \beta^{2}\right] A / M$.

Because $\beta, \gamma \in[0,1]$, we have $p_{1}^{* D C} \geq p_{2}^{* D C}$.

Similarly, from Theorem 1, we can also show that $q_{1}^{* D C} \leq q_{2}^{* D C}$.

Meanwhile, we can prove $\Pi_{S 1}^{* C D}>\Pi_{S 2}^{* C D}$ and $\Pi_{S 1}^{* D C}<\Pi_{S 2}^{* D C}$ by the similar method.

\section{Proof of Proposition 1:}

(i) From Theorem 1, we have

$$
\begin{aligned}
& \Pi_{S 2}^{* C C}-\Pi_{S 2}^{* C D} \\
& =\frac{A^{2}\left(4-\beta^{2}\right)}{4\left(\gamma^{2}+\beta^{2}-4\right)^{2}}-\frac{\left[4\left(2-\gamma^{2}\right)-\left(4-\gamma^{2}\right) \beta^{2}\right] A^{2}}{\left(4-\gamma^{2}\right)\left[4\left(2-\gamma^{2}\right)-2 \beta^{2}\right]^{2}} \\
& A^{2}\left(4-\beta^{2}\right)\left(4-\gamma^{2}\right)\left[4\left(2-\gamma^{2}\right)-2 \beta^{2}\right]^{2} \\
& =\frac{-4 A^{2}\left(\gamma^{2}+\beta^{2}-4\right)^{2}\left[4\left(2-\gamma^{2}\right)-\left(4-\gamma^{2}\right) \beta^{2}\right]}{4\left(4-\gamma^{2}\right)\left(\gamma^{2}+\beta^{2}-4\right)^{2}\left[4\left(2-\gamma^{2}\right)-2 \beta^{2}\right]^{2}} \text {. }
\end{aligned}
$$$$
\text { It is easy to find }
$$

$A^{2} /\left\{4\left(4-\gamma^{2}\right)\left(\gamma^{2}+\beta^{2}-4\right)^{2}\left[4\left(2-\gamma^{2}\right)-2 \beta^{2}\right]^{2}\right\}>$

0 . We assume

$f_{1}(\beta, \gamma)=4\left(4-\gamma^{2}\right)\left(2-\gamma^{2}\right)\left(4-3 \gamma^{2}\right)-(4-$ $\left.\gamma^{2}\right)\left(16-16 \gamma^{2}+3 \gamma^{4}\right) \beta^{2}+2\left(2-\gamma^{2}\right)^{2} \beta^{4}$.

Then there is $\Pi_{S 2}^{* C C}-\Pi_{S 2}^{* C D} \geq 0$ satisfied, if and only if the condition $f_{1}(\beta, \gamma) \geq 0$ is established. Here, we can regard $f_{1}(\beta, \gamma)$ as a quadratic function of $\beta^{2}$. Based on the properties of quadratic functions, we calculate

$\Delta=\left(4-\gamma^{2}\right)^{2}\left(16-16 \gamma^{2}+3 \gamma^{4}\right)^{2}-$ $32\left(2-\gamma^{2}\right)^{3}\left(4-\gamma^{2}\right)\left(4-3 \gamma^{2}\right)<0$

Finally, we can obtain $\Pi_{S 2}^{* C C} \geq \Pi_{S 2}^{* C D}$.

Similarly, we can prove $\Pi_{S 1}^{* C C} \geq \Pi_{S 1}^{* D C}$ and omit the details here.

(ii) From Theorem 1, we have

$\Pi_{S 1}^{* C D}-\Pi_{S 1}^{* D D}$

$=\varphi^{2} A^{2} /\left\{\left(4-\gamma^{2}\right)\left[4\left(2-\gamma^{2}\right)-2 \beta^{2}\right]\right\}^{2}$

$-\left(2-\gamma^{2}\right) \varphi^{2} A^{2} /\left\{\left(4-\gamma^{2}\right)\left[2 S T-\left(8-3 \gamma^{2}\right) \beta^{2}\right]^{2}\right\}$.
Let $Z_{1}\left(\beta_{1} \mid \gamma\right)=4\left(4-\gamma^{2}\right)^{1 / 2}\left(2-\gamma^{2}\right)^{3 / 2}-$ $2\left(4 \gamma^{4}-17 \gamma^{2}+16\right)+\left[8-3 \gamma^{2}-\right.$ $\left.2\left(4-\gamma^{2}\right)^{1 / 2}\left(2-\gamma^{2}\right)^{1 / 2}\right] \beta_{1}^{2}$.

Then, we find that $\Pi_{S 1}^{* C D}-\Pi_{S 1}^{* D D} \geq 0$ is equivalent to the condition of $Z_{1}\left(\beta_{1} \mid \gamma\right) \geq 0$.

(i) When $\beta_{1}=0$, we have $\partial Z_{1}(0 \mid \gamma) / \partial \gamma>0$, $Z_{1}(0 \mid 0)<0$ and $Z_{1}(0 \mid 1)>0$

(ii) When $\beta_{1}=1$, we have $\partial Z_{1}(1 \mid \gamma) / \partial \gamma>0$, $Z_{1}(1 \mid 0)<0$ and $Z_{1}(1 \mid 1)>0$.

Finally, we can obtain

if $(\gamma, \beta) \in\left\{0 \leq \gamma \leq \gamma_{1}, 0 \leq \beta \leq 1\right\}$

$\cup\left\{\gamma_{1} \leq \gamma \leq \gamma_{2}, 0 \leq \beta \leq \beta_{1}\right\}, \Pi_{S 1}^{* D D} \leq \Pi_{S 1}^{* C D}$;

if $(\gamma, \beta) \in\left\{\gamma_{1} \leq \gamma \leq \gamma_{2}, \beta_{1}<\beta \leq 1\right.$

$\cup\left\{\gamma_{2}<\gamma \leq 1,0 \leq \beta \leq 1\right\}, \Pi_{S 1}^{* D}>\Pi_{S 1}^{* C D}$, where the thresholds $\gamma_{1}(\approx 0.8062)$ and $\gamma_{2}(\approx 0.9309)$ are uniquely determined by the equations as $Z_{1}(0)$ $\left.\gamma_{1}\right)=0$ and $Z_{1}\left(1 \mid \gamma_{2}\right)=0$.

(iii) Similar to the proof of Part (ii), we can obtain the results in Part (iii).

Proof of Corollary 2:

(i) From Theorem 1, we have $\partial \Pi_{S 1}^{* C C} / \partial \beta^{2}=$ $A \gamma /\left[2\left(4-\gamma^{2}-\beta^{2}\right)^{2}\right]$. Because $\beta, \gamma \in[0,1]$, we have $\partial \Pi_{S 1}^{* C C} / \partial \beta^{2} \geq 0$.

Similarly, we can derive

$\partial \Pi_{S 2}^{* C C} / \partial \beta^{2}$

$=A\left(4+\gamma^{2}-\beta^{2}\right) /\left[4\left(4-\gamma^{2}-\beta^{2}\right)^{3}\right] \geq 0$.

(ii) From Theorem 1, we have

$\partial \Pi_{S 1}^{* D D} / \partial \beta^{2}$

$=\frac{2\left(2-\gamma^{2}\right)(\gamma-2) A^{2} \varphi}{\left(4-\gamma^{2}\right)\left[2 S T-\left(8-3 \gamma^{2}\right) \beta^{2}\right]^{2}}$

$-\frac{2\left(2-\gamma^{2}\right)\left(3 \gamma^{2}-8\right) A^{2} \varphi^{2}}{\left(4-\gamma^{2}\right)\left[2 S T-\left(8-3 \gamma^{2}\right) \beta^{2}\right]^{2}}$.

It is easy to find $2\left(2-\gamma^{2}\right) A^{2} \varphi /\left\{\left(4-\gamma^{2}\right)\left[2 S T-\left(8-3 \gamma^{2}\right) \beta^{2}\right]^{2}\right\}>$ 0 . Let $f_{2}(\beta, \gamma)=2\left(2 \gamma^{2}-4-\gamma\right)+(2-\gamma) \beta^{2}$.

Then, we find that $\partial \Pi_{S 1}^{* D D} / \partial \beta^{2}>0$ is equivalent to the condition of $f_{2}(\beta, \gamma)<0$. We can regard $f_{2}(\beta, \gamma)$ as a quadratic function of $\beta$.

(i) If $\beta=0, f_{2}(0, \gamma)=2\left(2 \gamma^{2}-4-\gamma\right)<0$;

(ii) If $\beta=1, f_{2}(1, \gamma)=2\left(2 \gamma^{2}-3-\gamma\right)-\gamma<0$.

Therefore we have $f_{2}(\beta, \gamma)<0, \forall \beta, \gamma \in[0,1]$, namely $\partial \Pi_{S 1}^{* D D} / \partial \beta^{2}>0$.

From Theorem 1, we have

$\partial \Pi_{S 2}^{* D D} / \partial \beta^{2}$

$=\frac{2\left(\gamma^{2}-4\right) A^{2} T^{2}}{\left(4-\gamma^{2}\right)\left[2 S T-\left(8-3 \gamma^{2}\right) \beta^{2}\right]^{2}}$

$-\frac{2\left(3 \gamma^{2}-8\right)\left[4\left(2-\gamma^{2}\right)-\left(4-\gamma^{2}\right) \beta^{2}\right] A^{2} \varphi^{2}}{\left(4-\gamma^{2}\right)\left[2 S T-\left(8-3 \gamma^{2}\right) \beta^{2}\right]^{2}}$. 
It is easy to find
$2 A^{2} /\left\{\left(4-\gamma^{2}\right)\left[2 S T-\left(8-3 \gamma^{2}\right) \beta^{2}\right]^{2}\right\}>0$. Let
$Z_{3}\left(\beta_{3} \mid \gamma\right)=2\left(\gamma^{2}-4\right) S T+8\left(8-3 \gamma^{2}\right)\left(2-\gamma^{2}\right)-$
$\left(4-\gamma^{2}\right)\left(8-3 \gamma^{2}\right) \beta_{3}^{2}$, and $\partial \Pi_{S 2}^{* D D} / \partial \beta^{2}>0$ is equiv-
alent to the condition of $Z_{3}\left(\beta_{3} \mid \gamma\right)>0$. Based on
the properties of quadratic functions, we have

(i) If $\beta_{3}=0, Z_{3}(0 \mid \gamma)=2 \gamma^{2}\left(4 \gamma^{4}-21 \gamma^{2}+28\right) \geq 0$;

(ii) If $\beta_{3}=1, Z_{3}(1 \mid \gamma)=8 \gamma^{6}-45 \gamma^{4}+76 \gamma^{2}-32$.

Next, we discuss $Z_{3}(1 \mid \gamma)$. We have $\partial Z_{3}(1 \mid \gamma) / \partial\left(\gamma^{2}\right)=24\left(\gamma^{2}\right)^{2}-90 \gamma^{2}+76>0$. Given $Z_{3}(1 \mid 0)=-32<0$ and $Z_{3}(1 \mid 1)=7>0$, $\exists \gamma_{5} \in[0,1]$, s.t. $Z_{3}(1 \mid \gamma)=0$.

Finally,

have

$\begin{cases}Z_{3}(1 \mid \gamma) \leq 0 & 0 \leq \gamma \leq \gamma_{5} \\ Z_{3}(1 \mid \gamma)>0 & \gamma_{5}<\gamma \leq 1\end{cases}$ and

$\begin{cases}Z_{3}\left(\beta_{3} \mid \gamma\right) \geq 0 & \text { if } 0 \leq \gamma \leq \gamma_{5}, 0 \leq \beta^{2} \leq \beta_{3}^{2} \\ Z_{3}\left(\beta_{3} \mid \gamma\right) \leq 0 & \text { if } 0 \leq \gamma \leq \gamma_{5}, \beta_{3}^{2}<\beta^{2} \leq 1 \\ Z_{3}\left(\beta_{3} \mid \gamma\right) \geq 0 & \text { if } \gamma_{5}<\gamma \leq 1, \forall \beta^{2} \in[0,1]\end{cases}$ where $\gamma_{5}(\approx 0.7933)$ is determined by $Z_{3}(1 \mid 0)=0$ and $\beta_{3}$ is determined by $Z_{3}\left(\beta_{3} \mid \gamma\right)=0$.

Proof of Theorem 2: Because the proof method of Theorem 2 is similar to that of Theorem 1, we omit the details here.

Proof of Corollary 3: Similar to the proof method of Corollary 3.

Proof of Proposition 2: Similar to the proof method of Proposition 2.

Proof of Corollary 4: Similar to the proof method of Corollary 4.

Proof of Proposition 3: Similar to the proof method of Proposition 3.

\section{Proof of Proposition 4:}

(i). From Theorems 1 and 2, we have

$\left(\tilde{e}_{2}^{* C C}\right)^{1 / 2}-\left(e_{2}^{* C C}\right)^{1 / 2}$ $A \beta /\left[(2+\gamma)\left(4-\beta^{2}-2 \gamma\right)\right]-A \beta /\left[2\left(4-\beta^{2}-\gamma^{2}\right)\right]$.

We find that the inequality of $\left(\tilde{e}_{2}^{* C C}\right)^{1 / 2} \geq$ $\left(e_{2}^{* C C}\right)^{1 / 2}$ is satisfied if and only if $2\left(4-\beta^{2}-\gamma^{2}\right)-$ $(2+\gamma)\left(4-\beta^{2}-2 \gamma\right) \geq 0$ is satisfied. Assuming $f_{6}(\beta, \gamma)=2\left(4-\beta^{2}-\gamma^{2}\right)-(2+\gamma)\left(4-\beta^{2}-2 \gamma\right)$, we find that $f_{6}(\beta, \gamma) \geq 0$ due to $\gamma, \beta \in[0,1]$, we have $\tilde{e}_{2}^{* C C} \geq e_{2}^{* C C}$.

Similarly, we can show other results. In the following, we give the comparisons on profits.

(ii) From Theorems 1 and 2, we have $\tilde{\Pi}_{S 2}^{* C C}-\Pi_{S 2}^{* C C}$

$=\left(4-\beta^{2}\right) A^{2} /\left[(2+\gamma)^{2}\left(4-\beta^{2}-2 \gamma\right)^{2}\right]$

$-A^{2}\left(4-\beta^{2}\right) /\left[4\left(\gamma^{2}+\beta^{2}-4\right)^{2}\right]$.

It is easy to find that the inequality of $\tilde{\Pi}_{S 2}^{* C C} \geq$ $\Pi_{S 2}^{* C C}$ is equivalent to $16-4 \gamma^{2}-4 \beta^{2}-\gamma \beta^{2} \geq$
0 , which is always satisfied. Therefore, we obtain $\tilde{\Pi}_{S 2}^{* C C} \geq \Pi_{S 2}^{* C C}$. Meanwhile, $\tilde{\Pi}_{S 2}^{* C C}=\Pi_{S 2}^{* C C}$ if and only if $\beta$ or $\gamma$ is 0 .

From Theorems 1 and 2, we find that the inequality of $\tilde{\Pi}_{S 2}^{* C D} \geq \Pi_{S 2}^{* C D}$ is equivalent to the condition of $8-4 \gamma^{2}+2 \gamma+(\gamma-2) \beta^{2} \geq 0$. Let $f_{7}(\beta, \gamma)=8-4 \gamma^{2}+2 \gamma+(\gamma-2) \beta^{2}$. Obviously, the function $f_{7}(\beta, \gamma)$ is a quadratic function of $\beta$.

Because $f_{7}(0, \gamma)>0$ and $f_{7}(1, \gamma)>0$, we can conclude that $f_{7}(\beta, \gamma)>0, \forall \gamma, \beta \in[0,1]$. Therefore, we have $\tilde{\Pi}_{S 2}^{* C D} \geq \Pi_{S 2}^{* C D}$ and $\tilde{\Pi}_{S 2}^{* C D}=\Pi_{S 2}^{* C D}$ if and only if $\beta$ or $\gamma$ is 0 . The proof of $\Pi_{S 2}^{* D C} \geq \Pi_{S 2}^{* D C}$ is similar to that of $\tilde{\Pi}_{S 2}^{* C D} \geq \Pi_{S 2}^{* C D}$ and we omit the details here.

From Theorems 1 and 2, we find that the inequality of $\tilde{\Pi}_{S 2}^{* D D} \geq \Pi_{S 2}^{* D D}$ is satisfied if and only if the condition of $2\left(2 \gamma^{2}-4+\gamma\right)\left(2 \gamma^{2}-4-\gamma\right)(3-$ $\left.\gamma^{2}\right)+(\gamma-3)\left(-\gamma^{3}-3 \gamma^{2}+3 \gamma+8\right) \beta^{2} \geq 0$ is satisfied.

We assume

$$
Z_{8}\left(\beta_{8} \mid \gamma\right)=2\left(2 \gamma^{2}-4+\gamma\right)\left(2 \gamma^{2}-4-\gamma\right)(3-
$$
$\left.\gamma^{2}\right)+(\gamma-3)\left(-\gamma^{3}-3 \gamma^{2}+3 \gamma+8\right) \beta_{8}^{2}$.

(i) When $\beta=0$, we have $Z_{8}(0 \mid \gamma)>0$;

(ii) When $\beta=1$, we have $Z_{8}(1 \mid \gamma)=-8 \gamma^{6}+$ $57 \gamma^{4}-122 \gamma^{2}-\gamma+72$.

Then we use MATLAB to calculate the solution of equation $-8 \gamma^{6}+57 \gamma^{4}-$ $122 \gamma^{2}-\gamma+72=0$ in $\gamma \in[0,1]$. The result is given by $\left\{\begin{array}{ll}Z_{8}(1 \mid \gamma) \geq 0 & 0 \leq \gamma \leq \gamma_{12} \\ Z_{8}(1 \mid \gamma)<0 & \gamma_{12}<\gamma \leq 1\end{array}\right.$, where $\gamma_{12} \approx 0.9705$. Finally, we obtain $\left\{\begin{array}{ll}Z_{8}\left(\beta_{8} \mid \gamma\right) \geq 0 & 0 \leq \gamma \leq \gamma_{12}, \forall \beta \in[0,1] \\ Z_{8}\left(\beta_{8} \mid \gamma\right) \geq 0 & \gamma_{12}<\gamma \leq 1,0 \leq \beta \leq \beta_{8} \\ Z_{8}\left(\beta_{8} \mid \gamma\right)<0 & \gamma_{12}<\gamma \leq 1, \beta_{8}<\beta \leq 1\end{array}\right.$, where the threshold $\beta_{8}$ is the unique solution determined by the equation as $Z_{8}\left(\beta_{8} \mid \gamma\right)=0$.

Proof of Proposition 5: From Propositions 1 and 2, we can obtain the main results of Proposition 5 .

Proof of Proposition 6: Here, comparisons of decision variables are obvious, and we omit them here. We only give the comparisons of profits.

From Theorem 1, we find that $\Pi_{S 2}^{* C C} \geq \Pi_{S 2}^{* D C}$ is satisfied if and only if the condition of $-2(2+$ $\gamma)+\beta^{2} \geq 0$ is satisfied. Because $\beta, \gamma \in[0,1]$, it is easy to prove $-2(2+\gamma)+\beta^{2}<0$. Therefore we have $\Pi_{S 2}^{* C C} \leq \Pi_{S 2}^{* D C}$. Similarly, we can prove that $\Pi_{S 2}^{* D D} \geq \Pi_{S 2}^{* C D}, \tilde{\Pi}_{S 2}^{* C C} \leq \tilde{\Pi}_{S 2}^{* D C}$ and $\tilde{\Pi}_{S 2}^{* D D} \geq \tilde{\Pi}_{S 2}^{* C D}$.

Proof of Proposition 7: (i) Here, the comparisons of decision variables will not be described in 
detail. The following discusses are mainly about comparisons of profits.

From Theorems 1 and 2, we find that $\tilde{\Pi}_{S 1}^{* D C} \geq$ $\Pi_{S 1}^{* C C}$ is satisfied if and only if the following condition is satisfied

$$
Z_{9}\left(\beta_{9} \mid \gamma\right)=2\left(4-\gamma^{2}\right)\left[4\left(2-\gamma^{2}\right)-\left(4-\gamma^{2}\right) \beta_{9}^{2}\right]
$$

$\left[\left(4-\gamma^{2}\right)-\beta_{9}^{2}\right]-\left[8\left(2-\gamma^{2}\right)\left(4-\gamma^{2}\right)-2\left(8-3 \gamma^{2}\right) \beta_{9}^{2}\right.$ $\left.-\left(4-\beta_{9}^{2}\right)\left(4-\gamma^{2}\right) \beta_{9}^{2}\right]^{2} \geq 0$.

Because $\partial^{2} Z_{9}\left(\beta_{9} \mid \gamma\right) /\left(\beta_{9}^{2}\right)^{2}<0$ and $\partial Z_{9}\left(\beta_{9} \mid \gamma\right) /\left(\beta_{9}^{2}\right)>0$, we find that $\partial Z_{9}\left(\beta_{9} \mid \gamma\right) /\left(\beta_{9}^{2}\right)$ is monotonically decreasing of $\beta_{9}^{2}$ and $Z_{9}\left(\beta_{9} \mid \gamma\right)$ is monotonically increasing of $\beta_{9}^{2}$. In addition, since $Z_{9}(0 \mid \gamma)<0$ and $Z_{9}(1 \mid \gamma)>0$, we have

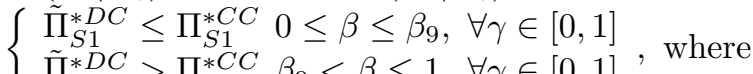
$\left\{\tilde{\Pi}_{S 1}^{* D C}>\Pi_{S 1}^{* C C} \beta_{9}<\beta \leq 1, \forall \gamma \in[0,1]\right.$, where

$\beta_{9}$ is the solution of equation as $Z_{9}\left(\beta_{9} \mid \gamma\right)=0$.

Based on Proposition 3 (i) and Proposition 5

(ii) (a), we can finally obtain Proposition 7 (i) (a).

In addition, we have $\tilde{\Pi}_{S 1}^{* C C} \geq \Pi_{S 1}^{* C C}$ and $\Pi_{S 1}^{* D C}<\Pi_{S 1}^{* C C}$ according to the Propositions 3 (i) and Proposition 5 (i) (a). Therefore, we have $\Pi_{S 1}^{* D C} \leq \tilde{\Pi}_{S 1}^{* C C}$.

(ii) Similar to the proofs of Part (i), we can obtain the results of part (ii).

Proof of Proposition 8: Similar to the proof method of Proposition 7.

\section{Compliance with Ethical Standards}

Ethical approval This article does not contain any studies with human participants or animals performed by any of the authors.

Conflict of interest The authors declare that they have no conflict of interest.

Informed Consent Informed consent was obtained from all individual participants included in the study.

\section{Authorship Contributions}

All authors contributed to the study conception and design. Material preparation and model analysis were performed by [Kebing Chen]. The first draft of the manuscript was written by [Mingxia Li] and all authors commented on previous versions of the manuscript. All authors read and approved the final manuscript.

\section{References}

Adida E, Demiguel V (2011) Supply chain competition with multiple manufacturers and retailers. Operations Research 59:156-172.

Ai X, Chen J, Ma J (2012) Contracting with demand uncertainty under supply chain competition. Annals of Operations Research 201:17-38.

Atkins D, Liang L (2010) A note on competitive supply chains with generalized supply costs. European Journal of Operational Research 207:13161320.

Cachon G, Harker P (2002) Competition and outsourcing with scale economies. Management Science 48:1314-1333.

Chen J, Guo Z (2014) Strategic sourcing in the presence of uncertain supply and retail competition. Production and Operations Management 23:1748-1760.

Chen K, Xiao T (2009) Demand disruption and coordination of the supply chain with a dominant retailer. European Journal of Operational Research 197:225-234.

Chen K, Xiao T, Wang S, et al (2020) Inventory strategies for perishable products with two-period shelf-life and lost sales. International Journal of Production Research 3:1-20.

CKGSB (2017) Will ecommerce replace brick-mortar-Chinese-retailers? http://kownledge.ckgsb.edu.cn /2017/02/27/retail/will-ecommerce-replacebrick-mortar-Chinese-retailers/.

Dan B, Liu C, Xu GY, Zhang XM (2014) Pareto improvement strategy for service-based freeriding in a dual-channel supply chain. AsiaPacific Journal of Operational Research 31: 1450050.

Desiraju R, Moorthy S (1997) Managing a distribution channel under asymmetric information with performance requirements. Management Science 43:1628-1644.

Ellram L, Tate W, Feitzinger E (2013) Factormarket rivalry and competition for supply chain resources. Journal of Supply Chain Management 49:29-46.

Fang Y, Shou B (2015) Managing supply uncertainty under supply chain Cournot competition. European Journal of Operational Research 243:156176.

Feng Q, Lu L (2013) Supply chain contracting under competition: Bilateral bargaining vs. Stackelberg. Production and Operations Management 22:661-675. 
Ghosh D, Shah J (2012) A comparative analysis of greening policies across supply chain structures. International Journal of Production Economics 135:568-583.

Ghosh D, Shah J (2014) Supply chain analysis under green sensitive consumer demand and cost sharing contract. International Journal of Production Economics 164:319-329.

Hosseinimotlagh S, Ebrahimi S, Jokar A, et al (2019) Sustainable supply chain coordination under competition and green effort scheme. Journal of the Operational Research Society Doi: 10.1080/01605682.2019.1671152.

Jamali M, Rasti-Barzoki M (2018) A game theoretic approach for green and non-green product pricing in chain-to-chain competitive sustainable and regular dual-channel supply chains. Journal of Cleaner Production 170:1029-1043.

Ke H, Jiang Y (2021) Equilibrium analysis of marketing strategies in supply chain with marketing efforts induced demand considering free riding. Soft Computing 25:2103-2103.

Lee H, Cruz J, Shankar R (2018) Corporate social responsibility (CSR) issues in supply chain competition: should greenwashing be regulated. Decision Sciences 49:1088-1115.

Li B, Zhou Y, Li J, et al (2013) Contract choice game of supply chain competition at both supplier and retailer levels. International Journal of Production Economics 143:188-197.

Li Q, Li B (2016) Dual-channel supply chain equilibrium problems regarding retail services and fairness concerns. Applied Mathematical Modelling 40:7349-7367.

Li ZH, Yang WS, Liu XH, Taimoor H (2020) Coordination strategies in dual-channel supply chain considering innovation investment and different game ability. Kybernetes 49: 1581-1603.

Liu Y, Wang D, Xu Q (2020) A supply chain coordination mechanism with suppliers' effort performance level and fairness concern. Journal of Retailing and Consumer Services 53: 101950.

Ma P, Li KW, Wang Z (2017) Pricing decisions in closed-loop supply chains with marketing effort and fairness concerns. International Journal of Production Research 55: 6710-6731.

McGuire T, Staelin R (1983) An industry equilibrium analysis of downstream vertical integration. Marketing science 2:161-191. (2008, reprinted)

Morris M, Schindehutte M, Allen J (2005) The entrepreneur's business model: toward a unified perspective. Journal of Business Research 58:726-735.
Song J, Li F, Wu D, Liang L, Dolgui A (2017) Supply chain coordination through integration of innovation effort and advertising support. Applied Mathematical Modelling 49: 108-123.

Wei J, Lu J, Zhao J (2020) Interactions of competing manufacturers' leader-follower relationship and sales format on online platforms. European Journal of Operational Research 280:508-522.

Wu C, Chen C, Hsieh C (2012) Competitive pricing decisions in a two-echelon supply chain with horizontal and vertical competition. International Journal of Production Economics 135:265-274.

Wu X, Zhou Y (2017) The optimal reverse channel choice under supply chain competition. European Journal of Operational Research 259:6366.

Xia Y, Xiao T, Zhang G (2016) The impact of product returns and retailer's service investment on manufacturer's channel strategies. Decision Sciences 48:918-955.

Xia Y, Xiao T, Zhang G (2019) Service investment and channel structure decisions in competing supply chains. Service Science 11:57-74.

Xiao T, Choi T, Cheng T (2014) Product variety and channel structure strategy for a retailerStackelberg supply chain. European Journal of Operational Research 233:114-124.

Yang F, Kong JJ, Jin MY (2019) Two-period pricing with selling effort in the presence of strategic customers. Asia-Pacific Journal of Operational Research 36:1950011.

Zhang Z, Xu H, Chen K (2021) Operational decisions and financing strategies in a capital-constrained closed-loop supply chain. International Journal of Production Research 59: 4690-4710.

Zhao X, Shi C (2011) Structuring and contracting in competing supply chains. International Journal of Production Economics 134:434-446.

Zhu W, He Y (2016) Green product design in supply chains under competition. European Journal of Operational Research 258:165-180. 